Aus der Abteilung Kardiologie und Pneumologie

(Prof. Dr. med. G. Hasenfuß)

im Zentrum Innere Medizin

der Medizinischen Fakultät der Universität Göttingen

\title{
Perkutan-transluminales Clipping bei Mitralklappeninsuffizienz
}

\author{
Inaugural-Dissertation \\ zur Erlangung des Doktorgrades \\ der Medizinischen Fakultät \\ der Georg-August-Universität zu Göttingen
}

\author{
vorgelegt von \\ Lars Berg \\ aus Hamburg.
}

Göttingen 2011 
Dekan:

I. Berichterstatter:

II. Berichterstatter/in:

III. Berichterstatter/in:
Prof. Dr. med. C. Frömmel

PD Dr. med. W. Schillinger

PD Dr. med. Tirilomis

PD Dr. med. Sigler

Tag der mündlichen Prüfung: 21.02.2012 


\section{Inhaltsverzeichnis}

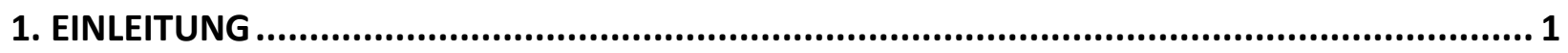

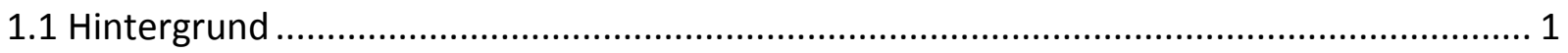

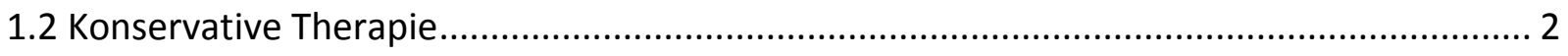

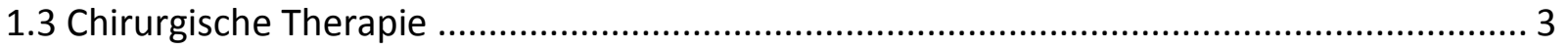

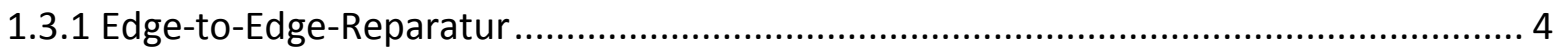

1.3.2 Morbidität und Mortalität der Mitralklappenchirurgie .......................................... 5

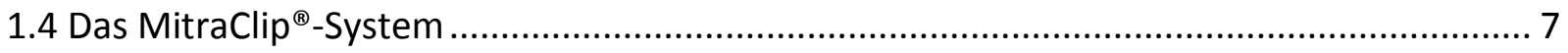

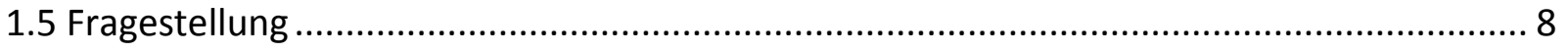

2. MATERIAL UND METHODIK................................................................................ 9

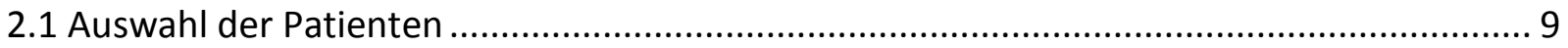

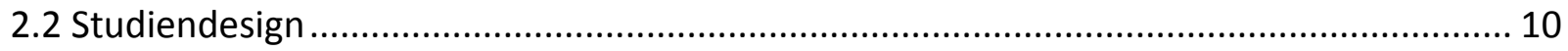

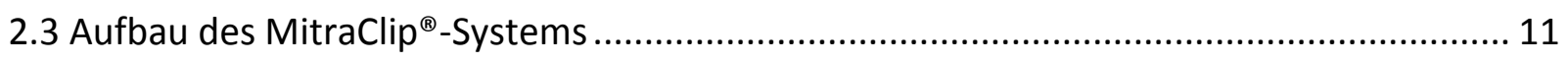

2.4 Ablauf des MitraClip ${ }^{\circledR}$-Eingriffes ................................................................................... 15

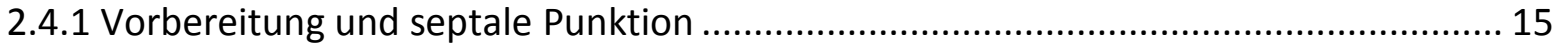

2.4.2 Positionierung des MitraClip ${ }^{\circledR}$-Systems............................................................... 17

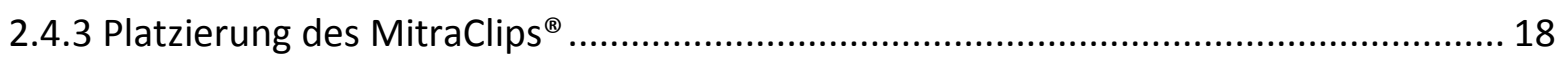

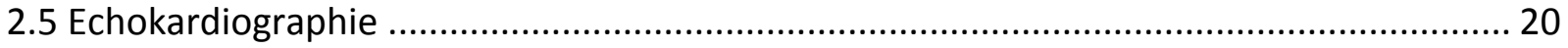

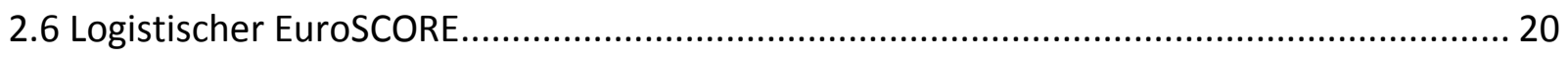

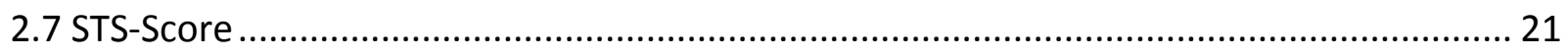

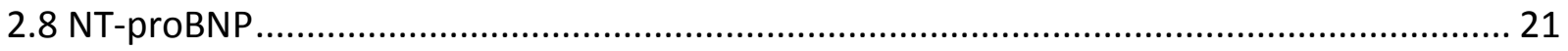

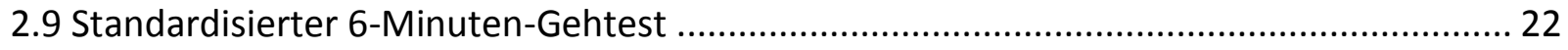

2.10 Minnesota Living With Heart Failure Questionnaire (MLHFQ) ................................... 22

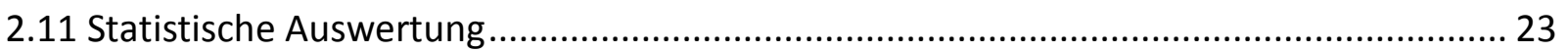

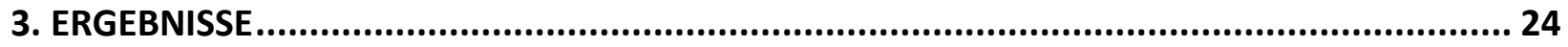

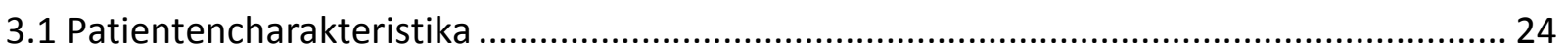

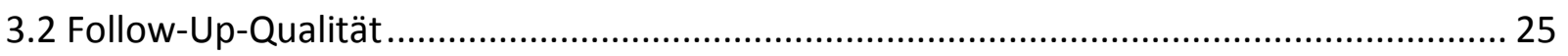

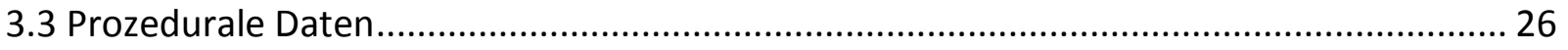

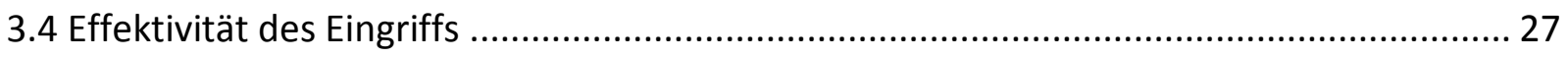

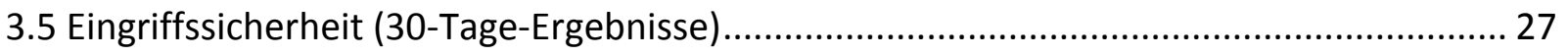

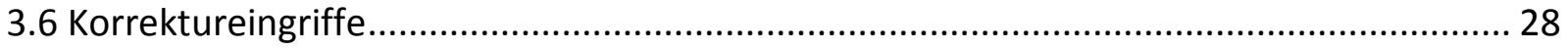

3.7 Beeinflussung des Mitralinsuffizienz-Grades über 12 Monate...................................... 29

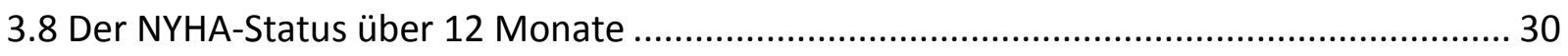

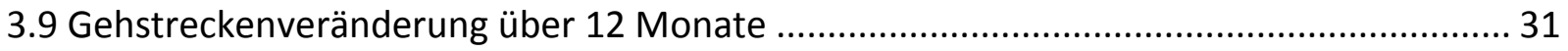

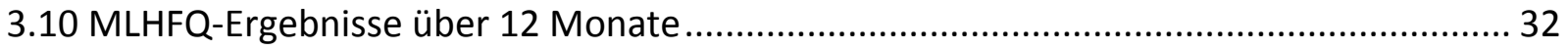


3.11 Reverses Remodeling durch das MitraClip ${ }^{\circledR}$-System ............................................... 33

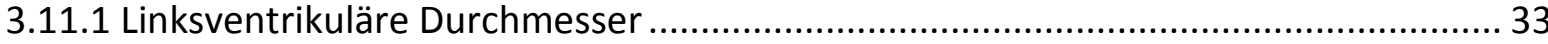

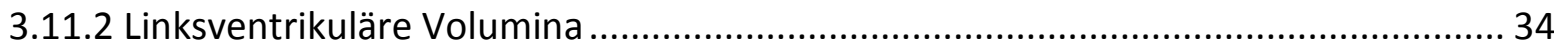

3.12 Ejektionsfraktion über 12 Monate ..................................................................... 36

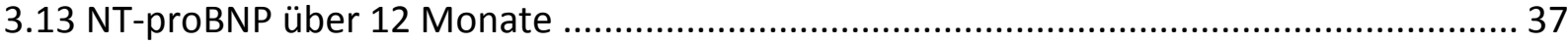

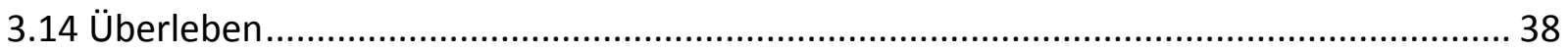

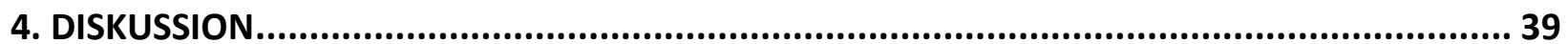

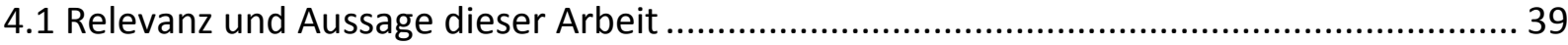

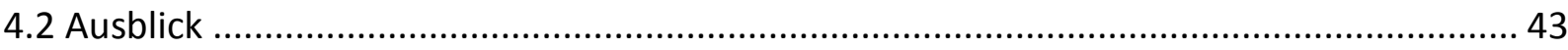

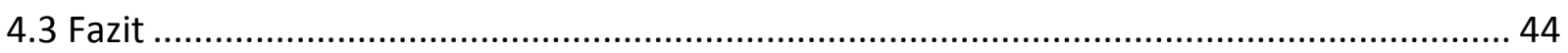

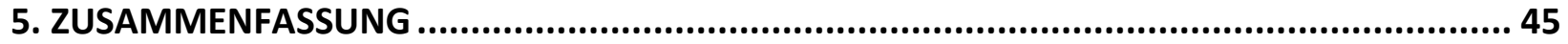

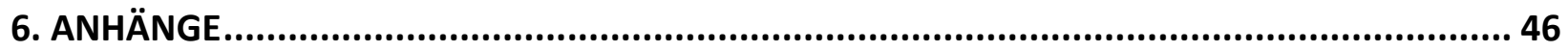

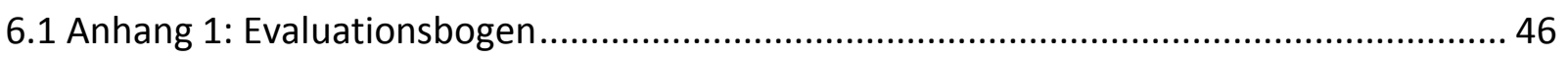

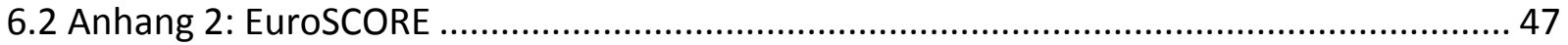

6.3 Anhang 3: Minnesota Living With Heart Failure Questionnaire (MLHFQ)........................ 48

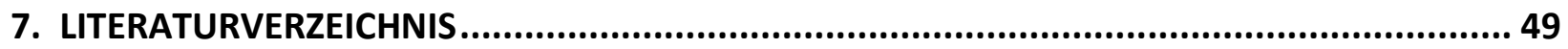




\section{Abkürzungsverzeichnis}

\begin{tabular}{|c|c|}
\hline ACC & American College of Cardiology \\
\hline ACT & Activated Clotting Time (Aktivierte Koagulationszeit) \\
\hline $\mathrm{AHA}$ & American Heart Association \\
\hline CDS & Clip Delivery System (Clip-Einführungssystem) \\
\hline COPD & Chronic Obstructive Pulmonary Disease (Chronisch obstruktive Lungenerkrankung) \\
\hline CRTD & Cardiac Resynchronization Therapy + Defibrillation \\
\hline EF & Ejektionsfraktion \\
\hline ESC & European Society of Cardiology (Europäische Gesellschaft für Kardiologie) \\
\hline EuroSCORE & European System for Cardiac Operative Risk Evaluation \\
\hline EVEREST & Endovascular Valve Edge-to-Edge Repair Study \\
\hline HZV & Herzzeitvolumen \\
\hline KHK & Koronare Herzkrankheit \\
\hline LA & Linkes Atrium \\
\hline LV & Linker Ventrikel \\
\hline LVEDD & Linksventrikulärer enddiastolischer Durchmesser \\
\hline LVEDV & Linksventrikuläres enddiastolisches Volumen \\
\hline LVEF & Linksventrikuläre Ejektionsfraktion \\
\hline LVESD & Linksventrikulärer endsystolischer Durchmesser \\
\hline LVESV & Linksventrikuläres endsystolisches Volumen \\
\hline LVOT & Left Venticular Outflow Tract (Linksventrikulärer Ausflusstrakt) \\
\hline MC & MitraClip ${ }^{\circledR}$ _Group (MitraClip ${ }^{\circledR}$-Therapiegruppe) \\
\hline MDRD & Modification of Diet in Renal Disease \\
\hline MI & Mitralklappeninsuffizienz \\
\hline
\end{tabular}




$\begin{array}{ll}\text { MK } & \text { Mitralklappe } \\ \text { MLHFQ } & \text { Minnesota Living With Heart Failure Questionnaire } \\ \text { MÖF } & \text { Mitralöffnungsfläche } \\ \text { MS } & \text { Mitralklappenstenose } \\ \text { 6MWT } & \text { 6-Minute-Walk-Test (6-Minuten-Gehtest) } \\ \text { NT-proBNP } & \text { N-terminal prohormone of brain natriuretic peptide } \\ \text { NYHA } & \text { New York Heart Association } \\ \text { pAVK } & \text { Periphere arterielle Verschlusskrankheit } \\ \text { SGC } & \text { Steerable Guide Catheter } \\ \text { STS } & \text { Society of Thoracic Surgeons } \\ \text { TEE } & \text { Transösophageale Echokardiographie } \\ \text { TTE } & \text { Transthorakale Echokardiographie } \\ \text { VHF } & \text { Vorhofflimmern }\end{array}$




\section{Einleitung}

\subsection{Hintergrund}

Mitralklappeninsuffizienz gehört zu den häufigsten Herzklappenfehlern. Nach der Europäischen Gesellschaft für Kardiologie (ESC) ist sie nach der degenerativen Aortenklappenstenose die zweithäufigste Herzklappenerkrankung. Viele Erkrankungen können zu einer Mitralinsuffizienz führen. Da rheumatische Klappenfehler aufgrund adäquater Therapie heutzutage kaum mehr eine Rolle spielen, ist das Alter von Patienten mit Mitralklappenerkrankungen mit im Mittel 65 Jahren nahezu so hoch wie das Alter von Patienten mit Aortenstenose, welches bei 69 Jahren liegt (Erbel et al. 2009).

Für das ordnungsgemäße Öffnen und Schließen der Mitralklappe ist die koordinierte Funktion von multiplen anatomischen Strukturen notwendig. Sowohl die Segel, der Anulus, die Chordae tendineae und Papillarmuskeln als auch der linke Vorhof und die linke Kammer des Herzens müssen optimal zusammenarbeiten, um eine effektive Mitralklappenfunktion ohne Stenose oder Insuffizienz zu ermöglichen. Die Funktionsstörung einer oder mehrerer dieser Strukturen führt entweder dazu, dass der Blutfluss durch die Klappe behindert wird (Mitralklappenstenose) oder dass die Klappensegel nicht richtig schließen und es als Folge zu einem Rückstrom des Blutvolumens in den linken Vorhof kommt (Mitralklappeninsuffizienz).

Patienten mit MI können über Jahre asymptomatisch sein. Um die einhergehende Volumenbelastung bewältigen zu können und das HZV aufrecht zu erhalten, tritt im Verlauf eine Vergrößerung des linken Ventrikels auf, was zu einer linksventrikulären Dysfunktion führt. Währenddessen dilatiert der linke Vorhof aufgrund des vermehrten Volumens, was zu Vorhofflimmern oder in einigen Fällen sogar zu Vorhofthromben und peripheren Embolisationen, z.B. einem Schlaganfall, führen kann.

Durch kompensatorische LA- und LV-Dilatation bleibt das HZV mitunter normal, was den Symptombeginn verzögern kann. Letzten Endes resultiert die chronische Volumenüberbelastung des LV in kontraktiler Dysfunktion, Symptomen der Herzinsuffizienz und einem erhöhten Risiko für einen plötzlichen Herztod. Bei Patienten mit hochgradiger symptomatischer MI liegt die jährliche Sterblichkeitsrate ohne chirurgische Intervention bei 5 \% (Grigioni et al. 1999; Rosen et al. 1994). Die häufigste Todesursache ist progredientes Herzversagen, aber auch plötzlicher Herztod, Apoplex und Endokarditis kommen als Todesursache vor. 
Die Ursachen der Mitralinsuffizienz sind unterschiedlich: Es wird zwischen organischer (degenerativer) sowie ischämischer und nicht-ischämischer funktioneller Mitralinsuffizienz unterschieden (Vahanian et al. 2007), wobei der Mitralinsuffizienz am häufigsten eine organische Ätiologie zugrunde liegt (lung et al. 2003). Die ischämische Herzkrankheit (KHK) und die nichtischämische Herzkrankheit (z.B. Kardiomyopathie) sind durch ventrikuläre Wandbewegungsstörungen, ventrikuläre Dilatation, Verlängerung oder Dysfunktion der Papillarmuskeln mit der funktionellen MI assoziiert. Degenerative MI ist durch strukturelle Klappenpathologie und pathologische Veränderungen des subvalvulären Apparates wie die Dehnung oder die Ruptur der Chordae tendineae charakterisiert.

\subsection{Konservative Therapie}

Bei Diagnosestellung der MI ist es in erster Linie wichtig, die Schwere und eine potentielle Reversibilität zu erkennen. Regelmäßige echokardiographische Kontrolluntersuchungen im Abstand von 6 Monaten bis zu 5 Jahren werden von den ACC/AHA-Richtlinien je nach Grad der MI, der Ejektionsfraktion und des LVESD empfohlen (Otto 2001).

Allerdings haben die meisten Patienten bei Diagnosestellung eine leicht- bis mittelgradige $\mathrm{MI}$, bei welcher kein operativer Eingriff, sondern eine konservative Therapie eingeleitet wird (Bonow et al. 1998). Es gibt allerdings keine Langzeitstudien, welche eine Reduktion der Mortalität bei pharmakologischer konservativer Therapie zeigen. Durch die Gabe von Nachlast-senkenden Medikamenten soll der retrograde Fluss verringert werden, was wiederum zu einer Zunahme des antegraden Flusses führt und damit die Dilatation des LV verzögert. Obwohl bei akuter $\mathrm{MI}$ eine Nachlast-Senkung die Schwere der mitralen Regurgitation reduziert und die Hämodynamik günstig beeinflusst (Vahanian et al. 2007), gibt es bei chronischer MI keine Studie, die zeigt, dass eine optimale Nachlastsenkung die Notwendigkeit für eine operative Therapie hinauszögert oder die Mortalität reduziert. Einige Studien haben eine vasodilatative Therapie zur Besserung der Symptome, jedoch nicht zur Besserung der LV-Funktion vorgeschlagen. Entsprechend den ACC- und AHA-Richtlinien von 1998 gibt es keine Indikation für die Gabe von vasodilatativen Medikamenten bei asymptomatischen Patienten mit gut erhaltener LV-Funktion, solange keine systemische Hypertonie vorliegt (Otto 2001). 


\subsection{Chirurgische Therapie}

Bei Patienten mit symptomatischer hochgradiger MI oder asymptomatischer hochgradiger MI mit Nachweis einer LV-Dysfunktion oder Dilatation wird heutzutage eine chirurgische Therapie empfohlen (Bonow et al. 1998). Mitralklappenchirurgie (Reparatur oder Ersatz) ist nach den Zahlen der „Society of Thoracic Surgeons (STS) Database“ die zweithäufigste Herzklappenoperation in den USA (STS-Database 1997).

Die Diagnose MI wurde allein 1995 in den USA mehr als 500.000 mal gestellt und es wurden mehr als 30.000 Mitralklappenoperationen durchgeführt (Graves und Owings 1997).

Gegenwärtig ist die einzige erprobte Therapie für hochgradige MI die offene, unter extrakorporaler Zirkulation durchgeführte Klappenreparatur oder der Klappenersatz. Sowohl der Mitralklappenersatz als auch die Rekonstruktion der Mitralklappe haben ein geringes operatives Risiko. Zu den Risiken gehören neben den Risiken des chirurgischen Eingriffes selbst sowohl kardiologische, neurologische, respiratorische und renale Komplikationen als auch deren Folgen. Einige dieser Komplikationen sind mit der extrakorporalen Zirkulation verbunden. Zusätzlich benötigen die meisten Patienten nach der Operation eine längere Erholungsphase. Obwohl die Resultate der Operation bezüglich der Mitralinsuffizienz sehr gut sind, wird sie für einige Patienten erst verspätet durchgeführt oder ist aufgrund von Morbidität oder hohem operativem Risiko keine Option. Wenn eine Klappenreparatur technisch möglich ist, bietet sie Vorteile gegenüber dem Klappenersatz. Das belegt ein Vergleich der Ergebnisse bezüglich des klinischen Resultates, der LVFunktion, der Mortalität und der Vermeidung von Langzeitantikoagulation (Enriquez-Sarano et al. 1995). Nach Zahlen verschiedener Quellen (u.a. "Healthcare Costs and Utilization Project 2002", "Nationwide Inpatient Sample (NIS)", "Agency for Healthcare Research and Quality (AHRQ)", der "2002 STS Database" und Daten des "Columbia Presbyterian Hospital") lag das durchschnittliche Verhältnis von Mitralklappenreparatur zu Mitralklappenersatz vor 2002 in den USA bei 40 \% zu 60 \%. Neuere Zahlen zeigen eine Zunahme der Mitralklappenreparatur im Verhältnis zum Mitralklappenersatz. In den meisten Zentren in Europa und den USA sind inzwischen mindestens 50 \%, in erfahrenen Zentren bis 90 \% der Eingriffe Mitralklappenreparaturen (Enriquez-Sarano et al. 2003; lung et al. 2003). Die Reparaturrate hängt von der Art der Läsion ab: Bei einem isolierten Prolaps des posterioren Segels ist die Anzahl der Reparaturen in den meisten Zentren hoch, während eine Reparatur des anterioren Segels, bei Kalzifikationen und bei einer rheumatischen Beteiligung weniger häufig durchgeführt wird (Bonow et al. 1998). 
Reparaturtechniken schließen die verhältnismäßig komplexe Resektion, Reposition und Umgestaltung der Segel ein. Die Reparatur des anterioren Segels ist besonders komplex, da auch ein Eingriff an den Chordae tendineae notwendig sein kann, um die MI zu beseitigen. Aktuelle Reparaturtechniken für die Mitralklappe, einschließlich der Anuloplastie, wurden ursprünglich für die degenerative $\mathrm{MI}$ entwickelt und können für andere Ursachen der MI unangebracht sein. Diese Reparaturtechniken wurden sowohl mit als auch ohne Anuloplastiering durchgeführt.

\subsubsection{Edge-to-Edge-Reparatur}

Seit Anfang der 1990er Jahre wird die Edge-to-Edge-Reparatur als Behandlung der MI eingesetzt. Sie wurde als offene Operation unter extrakorporaler Zirkulation durchgeführt. Bei dieser Technik wird durch eine Naht ein Teil des anterioren Segels an den entsprechenden Teil des posterioren Segels genäht, wodurch an dieser Stelle eine permanente Koaptation der beiden Segel entsteht. Wenn die Edge-to-Edge-Naht in der Mitte der Klappe gesetzt wird, entsteht während der Diastole ein sogenanntes funktionelles „double-orifice“, also eine doppelte Öffnung beidseits der Naht. Daher nennt man diesen Eingriff auch „Double-Orifice-Reparatur" (siehe Abb.1). Die Klappe öffnet sich immer noch an beiden Seiten der Naht, was ausreichenden diastolischen Blutfluss durch die Klappe erlaubt. Die Annäherung der beiden Segel und der mit der Zeit einsetzende Heilungsprozess entlastet die Naht. Es wurde in der Fachliteratur von über 1000 offenen Operationen mit der Edgeto-Edge-Technik mit Follow-Up-Untersuchungen von bis zu 9 Jahren berichtet. Die Mortalität dieser Technik unterscheidet sich nicht signifikant von der Mortalität der öfter durchgeführten StandardReparatur-Techniken (Alfieri und Maisano 1999; Maisano et al. 1998; Maisano et al. 2000; Umana et al. 1998). Diese „Double-Orifice-Reparatur" nach Alfieri wurde erfolgreich zur Behandlung aller primären Ätiologien der Ml eingesetzt. Edge-to-Edge-Reparatur wird bei offenen Rekonstruktionen selten und nur bei einem Teil der Patienten eingesetzt. Die neue Geometrie der Klappe bei Edge-toEdge-Reparatur hat eine kleinere diastolische MÖF als vor dem Eingriff zur Folge, genauso wie es bei der Anuloplastie oder dem Mitralklappenersatz der Fall ist. Eine typische Mitralklappe hat eine MÖF von 6-8 $\mathrm{cm}^{2}$. Die Egde-to-Edge-Technik reduziert die effektive MÖF im Durchschnitt um 40-50 \%, was einer Fläche von $3 \mathrm{~cm}^{2}$ oder mehr entspricht (Maisano et al. 2000). Klinisch signifikante Stenosen der Mitralklappe beginnen erst bei einer MÖF von unter $1,5 \mathrm{~cm}^{2}$. Mehrere Publikationen haben gezeigt, dass diese Technik keinen signifikanten diastolischen Druckgradienten 
über die Klappe erzeugt und auch über längere Zeit nicht zu einer MS führt (Lawrie 1998; Maisano et al. 1998; Maisano et al. 2000; Umana et al. 1998).

Es gibt sowohl in veröffentlichten als auch in unveröffentlichten Arbeiten Erkenntnisse in der Mitralklappenreparatur mit Edge-to-Edge-Technik ohne Anuloplastie, jedoch wurde in der Mehrheit der berichteten Fälle auch eine Anuloplastie durchgeführt. Viele Chirurgen führen eine Anuloplastie bei der Erstreparatur der MK durch, um das Risiko einer erneuten Operation in der Zukunft zu vermeiden. Wie weiter unten beschrieben steigen die Morbidität und Mortalität signifikant, wenn eine zweite offene, unter extrakorporaler Zirkulation durchgeführte Operation durchgeführt wird. Es gibt Hinweise dafür, dass die alleinige Edge-to-Edge-Reparatur den Spinktermechanismus der MK und somit die systolische Leistung der Herzbasis erhält.

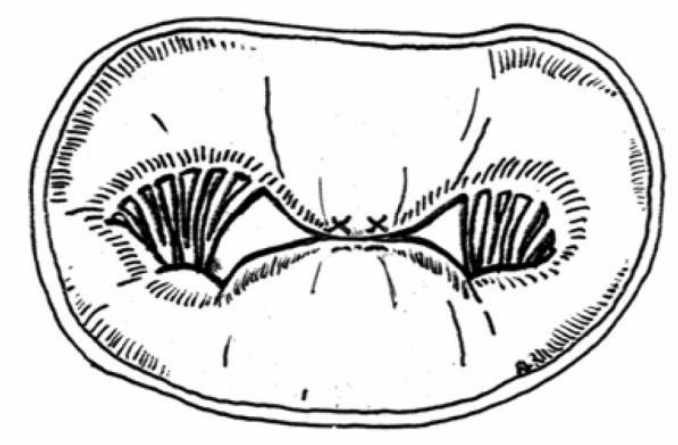

Abb. 1: Edge-to-Edge-Reparatur ((Maisano et al. 1998) Seite 242)

\subsubsection{Morbidität und Mortalität der Mitralklappenchirurgie}

Tab. 1 zeigt die Morbidität und Mortalität des Mitralklappenersatzes und der Mitralklappenreparatur bei Ersteingriff während des postoperativen Krankenhausaufenthaltes bis zu 30 Tagen (STS-Database 1997). Die Morbidität und Mortalität können bei bestimmten Patientenuntergruppen mit MI wesentlich höher sein. Die 30-Tage-Mortalität, gemittelt aus 15 chirurgischen Reparaturstudien (Akins et al. 1994; Alvarez et al. 1996; Carpentier 1983; Cohn et al. 
1994; Enriquez-Sarano et al. 1995; Fucci et al. 1995; Galloway et al. 1989; Gillinov et al. 1998; Gillinov et al. 2001; Grossi et al. 2001; Lawrie 1998; Lorusso et al. 2001; Maisano et al. 1998; Maisano et al. 2000; Totaro et al. 1999) betrug $3.75 \%$ und ist somit etwas höher als die Daten aus der STS-Datenbank für die chirurgische Mitralklappenreparatur. Die Rate an Frühkomplikationen ist mit der der STS-Datenbank vergleichbar. Die Mortalität von Patienten mit einer ischämischen Ätiologie ist weitaus höher und erreicht 10-13 \% (Gillinov et al. 2001; Grossi et al. 2001). Sowohl in den oben genannten Studien als auch in der STS-Datenbank ist die Gesamtmorbidität bei wiederholter Operation signifikant höher. Aus diesem Grund benutzen Chirurgen alle verfügbaren und angemessenen Reparaturtechniken während des Ersteingriffes, um damit das Risiko von Reoperationen zu verringern.

\begin{tabular}{|l|c|c|}
\hline \multicolumn{1}{|c|}{ Komplikation } & Mitralklappenreparatur & Mitralklappenersatz \\
\hline Tod & $1.5 \%$ & $6.0 \%$ \\
\hline Apoplex & $1.2 \%$ & $2.0 \%$ \\
\hline Nierenversagen & $2.5 \%$ & $5.1 \%$ \\
\hline Beatmung >24h & $5.0 \%$ & $12.9 \%$ \\
\hline Myokardinfarkt & $0.3 \%$ & $0.4 \%$ \\
\hline Herzbeuteltamponade & $1.0 \%$ & $2.4 \%$ \\
\hline $\begin{array}{l}\text { Reoperation } \\
\text { Blutung Klappendysfunktion } \\
\text { Andere kardiologische } \\
\text { Komplikation }\end{array}$ & $3.2 \%$ & $5.1 \%$ \\
\hline Sepsis & $0.6 \%$ & $0.5 \%$ \\
\hline Tiefe Wundinfektion & $2.2 \%$ & $3.7 \%$ \\
\hline $\begin{array}{l}\text { Gastrointestinale Blutung oder } \\
\text { Infarkt }\end{array}$ & $1.0 \%$ & $2.4 \%$ \\
\hline Neues Auftreten von VHF & $0.2 \%$ & $0.3 \%$ \\
\hline
\end{tabular}

Tab. 1: : Morbidität und Mortalität der isolierten Mitralklappenchirurgie bei Ersteingriff während des postoperativen Krankenhausaufenthalts bis 30 Tagen

(STS-Database 1997) 


\subsection{Das MitraClip ${ }^{\circledR}$-System}

In Zusammenarbeit mit interventionellen Kardiologen und Kardiochirurgen hat die Firma Abbott Vascular ein System entwickelt, das eine Edge-to-Edge-Rekonstruktion am schlagendem Herzen als Alternative zur offenen, mit extrakorporaler Zirkulation durchgeführten Operation ermöglicht. Das MitraClip ${ }^{\circledR}$-System von Abbott Vascular beinhaltet ein “Clip Delivery System" (CDS), welches das Platzieren eines Clips an die Mitralklappensegel erlaubt, was zu einer permanenten Koaptation der Segel führt. Dieses Verfahren basiert auf der Edge-to-Edge-Nahttechnik von Alfieri. Der Abstand der beiden Segel an der Koaptationsstelle ist durch die Implantation eines oder mehrerer Clips mit dem des Edge-to-Edge-Verfahrens vergleichbar. Die Vorteile des MitraClip ${ }^{\circledR}$-Systems, welche in der EVEREST-I-Studie beobachtet wurden, sind vor allem das Vermeiden der Thorakotomie, der extrakorporalen Zirkulation und der Kardioplegie bei den Patienten. Ein weiterer Vorteil des Gerätes besteht darin, das Ergebnis der Mitralklappenreparatur bei voll funktionstüchtigem Herzen vor Abschluss des Eingriffes beurteilen zu können. Es wurde darauf hingewiesen, dass sich der optimale Ort für eine Edge-to-Edge-Reparatur der Segel am besten bei schlagendem Herzen beurteilen lässt (Kron et al. 2002).

Mit dem MitraClip ${ }^{\circledR}$-System ist es möglich, die Reduktion der MI zu optimieren, indem man den Clip an verschiedenen Stellen der Koaptationslinie platziert und dann direkt echokardiographisch den Grad der MI evaluiert. Zusätzlich ist es mit dem MitraClip ${ }^{\circledR}$-System möglich, alle Schritte bis zum Ablösen des Clips jederzeit rückgängig zu machen. Wenn der Arzt mit dem Ergebnis der ersten Platzierung des Clips nach Erfassung und Annäherung der Segel nicht zufrieden ist, kann das Gewebe ohne Schäden losgelassen werden und derselbe Clip kann repositioniert oder sogar entfernt werden, ohne dass die Segel oder der subvalvuläre Apparat beschädigt werden. Sollte es nicht möglich sein, den Clip an der vorgesehenen Stelle zu platzieren, um die MI ausreichend zu reduzieren, kann der Clip komplett entfernt werden und der Patient hat nach wie vor die gleichen chirurgischen Optionen wie vor dem Versuch, die Mitralklappe mit dem MitraClip ${ }^{\circledR}$-System zu rekonstruieren (Rogers et al. 2009). Des Weiteren ist es möglich, nach einer erfolgreichen Implantation eines Clips weitere Clips zu platzieren, wenn dies eine weitere Reduktion der MI ergeben würde. 


\subsection{Fragestellung}

Die vorliegende Arbeit untersuchte Sicherheit und Effektivität des MitraClip-Systems bei den ersten 36 Patienten der Universitätsmedizin Göttingen, die aus klinischer Indikation der kathetergestützten Mitralklappenrekonstruktion mit MitraClip unterzogen wurden und über mindestens 12 Monate nachbeobachtet wurden. Unter anderem wird die Frage geprüft, wie sich das Verfahren im Verlauf über 12 Monate auf den Grad der Mitralinsuffizienz und die NYHA-Klasse auswirkt. Die körperliche Leistungsfähigkeit wird außerdem anhand des standardisierten 6Minuten-Gehtests über 12 Monate gemessen, um einen eventuellen Leistungszuwachs nachzuweisen. Mit dem MLHFQ soll die Lebensqualität erfasst und im Verlauf dokumentiert werden. Weiterhin wird mit Hilfe der transthorakalen Echokardiographie in den Nachsorgeuntersuchungen festgestellt, ob ein reverses Remodeling des Herzens stattgefunden hat. Dies wird sowohl durch die linksventrikulären Durchmesser, die linksventrikulären Volumina als auch durch die Erfassung der Ejektionsfraktion dokumentiert. Als Herzinsuffizienzparameter wurde das NT-proBNP im Verlauf bestimmt. Abschließend soll die Frage diskutiert werden, ob das MitraClip ${ }^{\circledR}$-System in Ergänzung zu konventionellen Methoden als sinnvolle zusätzliche Therapieoption, z.B. bei Patienten mit erhöhtem operativem Risiko, eingesetzt werden kann. 


\section{Material und Methodik}

\subsection{Auswahl der Patienten}

Patienten mit symptomatischer MI wurden einem intensiven Screening unterworfen, welches neben der klinischen Untersuchung, einer ausführlichen Anamnese zu Symptomen, Vorerkrankungen und Risiken und der Laborwerte jeweils ein TTE, ein TEE, die Berechnung des logistischen EuroScores, des STS-Scores und eine eventuelle Koronarangiographie bei V.a. KHK beinhaltete. Die Indikationsstellung erfolgte durch das multidisziplinäre Heart Team des Herzzentrums Göttingen unter Berücksichtigung aktueller Leitlinien (Vahanian et al. 2007), des operativen Risikos, der Gesamtprognose, anatomischer Aspekte und der Erfolgschancen des Eingriffs. Einschluss und Behandlung erfolgten von April 2009 bis März 2010. Die Follow-UpUntersuchungen wurden bis März 2011 vorgenommen. Abbildung 2.1 zeigt den Einschlussweg für die Patientenselektion:

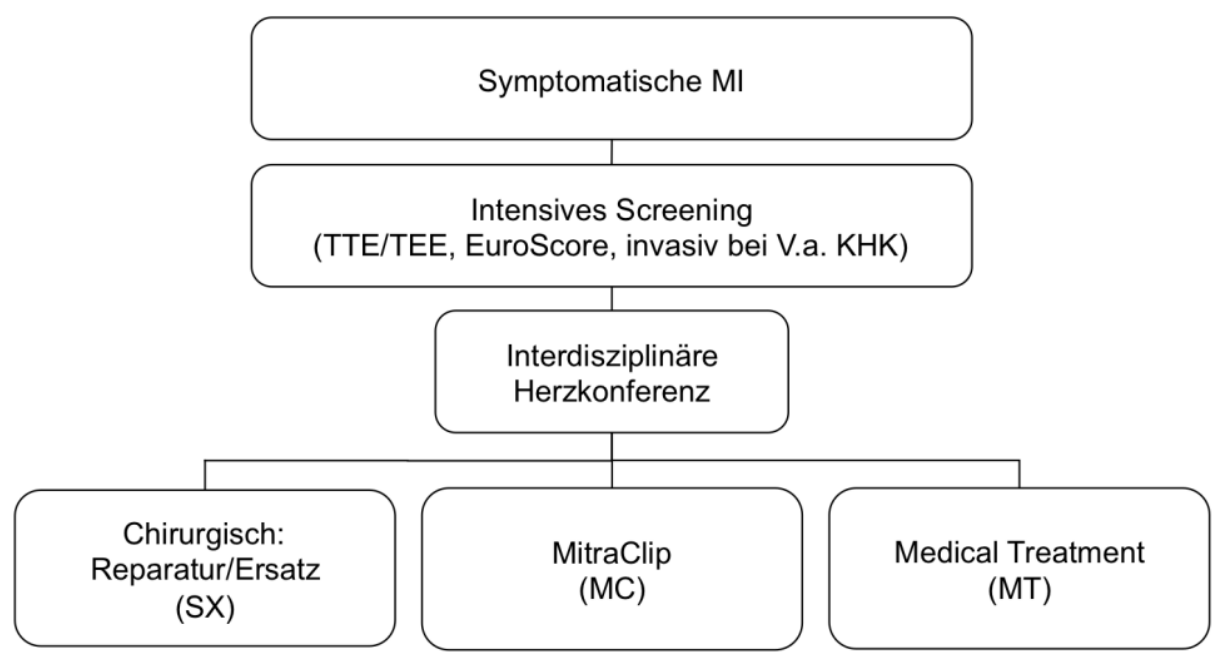

Abb. 2.1: Diagramm der Patientenauswahl

Nach eingehendem Screening wurde ein Evaluationsbogen ausgefüllt, welcher die Grundlage der Entscheidung in der interdisziplinären Herzkonferenz darstellte (siehe Anhang 1).

Nach der Entscheidung der Therapieoption wurden die Patienten in der MC-Gruppe über die Studie aufgeklärt und nach Zustimmung in die Studie eingeschlossen. 


\subsection{Studiendesign}

Die durchgeführte Studie war eine prospektive Beobachtungsstudie zur Evaluation der ersten 36 konsekutiven Patienten, die am Herzzentrum der Universitätsmedizin Göttingen einer perkutanen Mitralklappen-Reparatur mit dem MitraClip ${ }^{\circledR}$-System (Abbott Vascular) unterzogen wurden. Diese 36 Patienten wurden daraufhin über 12 Monate beobachtet. Es lagen ein positives Votum der Ethikkommission der Universitätsmedizin Göttingen und die schriftliche Einwilligung der Patienten für die wissenschaftliche Verwendung der Daten vor. Die Patienten wurden zu 3 Zeitpunkten untersucht: Jeweils vor dem Eingriff, nach 6 Monaten und nach 12 Monaten. Die Patienten wurden dazu zu Kontrolluntersuchungen in die Universitätsmedizin Göttingen einbestellt. Zwischenzeitlich eingetretene Ereignisse wurden dokumentiert. Nach der Aufklärung und dem Studieneinschluss der Patienten erfolgte die klinische Untersuchung, die Durchführung des MLHFQ, der standardisierte 6MWT, die Bestimmung des NT-proBNP sowie ein TTE, wobei der Schweregrad der Mitralinsuffizienz, die LVEDV, LVESV, LVEDD, LVESD und die EF dokumentiert wurden. Bei allen Patienten wurde außerdem der NYHA-Status erhoben. Im Rahmen der Kontrolluntersuchungen nach 6 Monaten und 12 Monaten wurde erneut ein TTE mit Quantifizierung der MI, LVEDV, LVEDS, LVEDD, LVESD, EF-Berechnung, das NT-proBNP im Serum, der NYHA-Status, der MLHFQ und der standardisierte 6MWT durchgeführt. Eventuelle zwischenzeitliche Hospitalisationen aus kardiologischen Gründen seit Studieneinschluss wurden ebenfalls dokumentiert.

Als schwere kardiovaskuläre Ereignisse wurden folgende Komplikationen erfasst: Tod, Hospitalisierung wegen Herzinsuffizienz, schwere Blutung nach GUSTO (GUSTO-Investigators 1993), Myokardinfarkt, Schlaganfall, kardiovaskuläre Operation, mechanische Kreislaufunterstützung, verlängerte Beatmungsdauer (>24h), Phlebothrombose, akutes Nierenversagen und partielle oder vollständige Cliplösung bzw. -embolisation. Mortalität, Hospitalisation und weitere Ereignisse wurden darüber hinaus unmittelbar vor Fertigstellung der Arbeit telefonisch erfragt und dokumentiert. 


\subsection{Aufbau des MitraClip ${ }^{\circledR}$-Systems}

Das MitraClip ${ }^{\circledR}$-System ist ein kathetergestützes System zur Edge-to-Edge-Rekonstuktion der insuffizienten Mitralklappe bei schlagendem Herzen als Alternative zu einer konventionellen Operation. Das MitraClip ${ }^{\circledR}$-System besteht aus einem steuerbaren Führungskatheter (Steerable Guide Catheter $=\mathrm{SGC}$ ), einem Clip-Einführungssystem $($ Clip Delivery System $=\mathrm{CDS})$ und dem Clip selbst. Das CDS ermöglicht die genaue Platzierung des Clips an die Mitralsegel, sodass diese permanent aneinander haften und beidseits des Clips ein Öffnung entsteht (sog. Double Orifice). Der Clip ist an der Spitze des CDS vormontiert.

Durch Steuerung der Führungsmechanismen am SGC und CDS wird eine exakte Positionierung über der Mitralklappe ermöglicht. Über die Hebel des CDS kann man den Clip öffnen, schließen, verriegeln und loslösen.

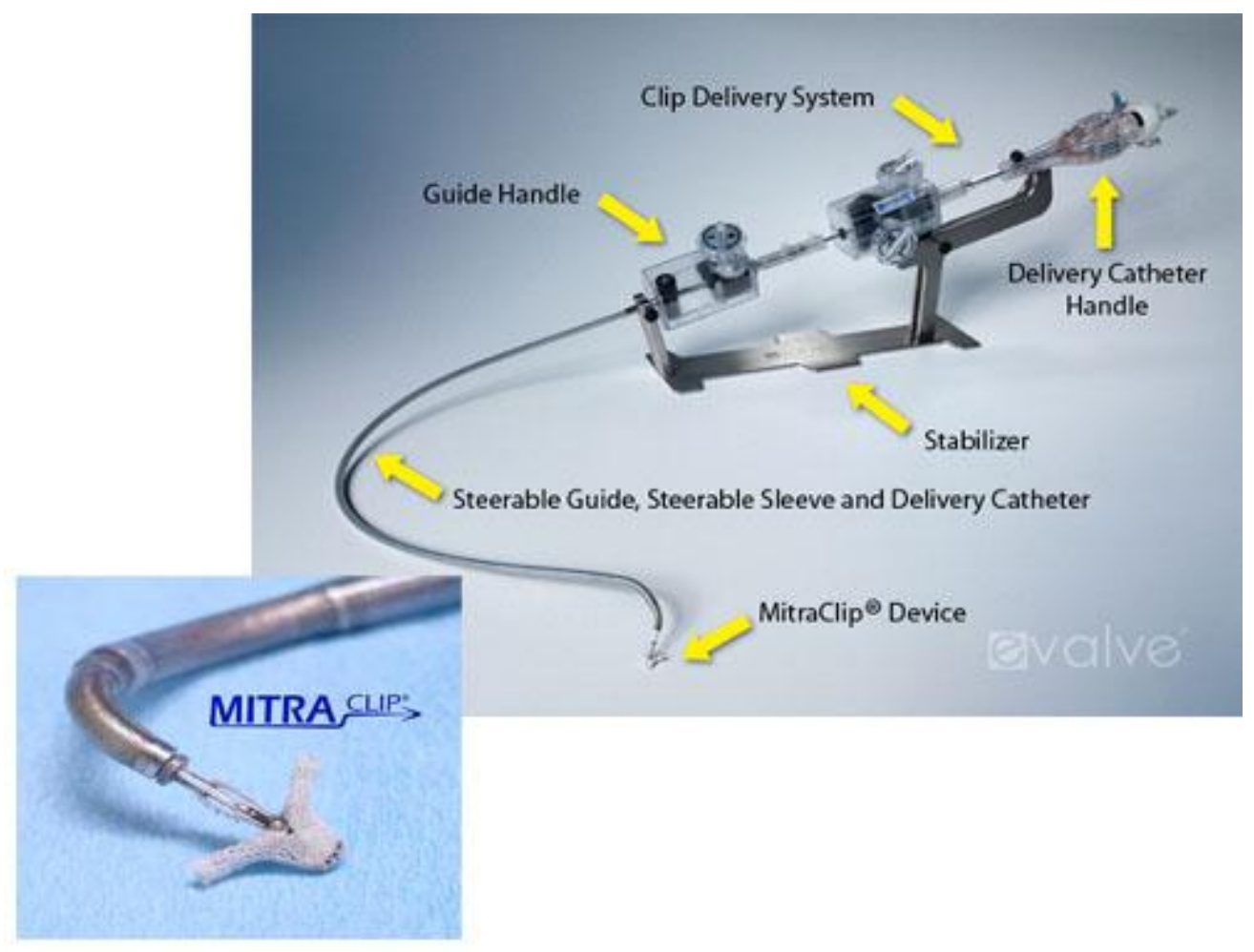

Abb. 2.2: Das MitraClip ${ }^{\circledR}$-System

(Abbott-Vascular 2008) 


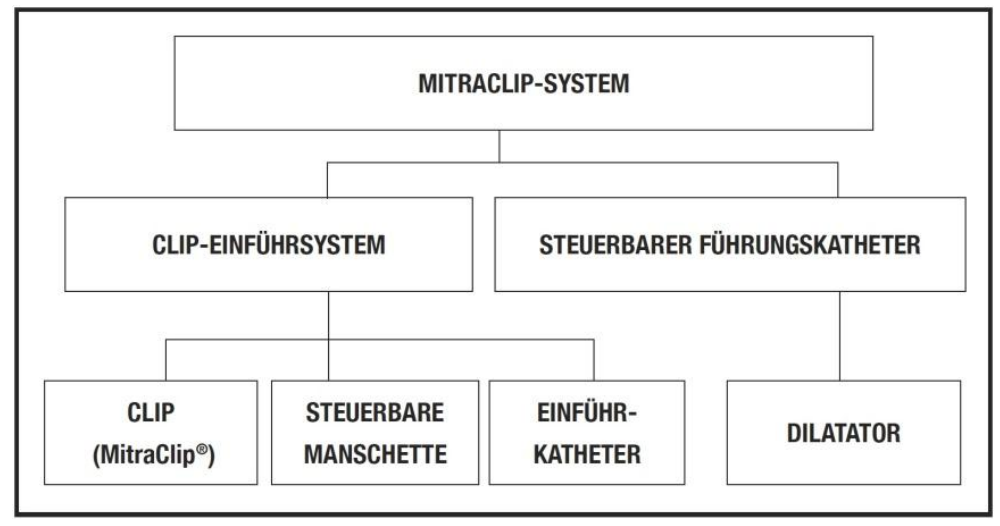

Abb. 2.3: Aufbau des MitraClip ${ }^{\circledR}$-Systems

(Abbott-Vascular 2008) 
Steuerbarer Führungskatheter:

Dilatator:

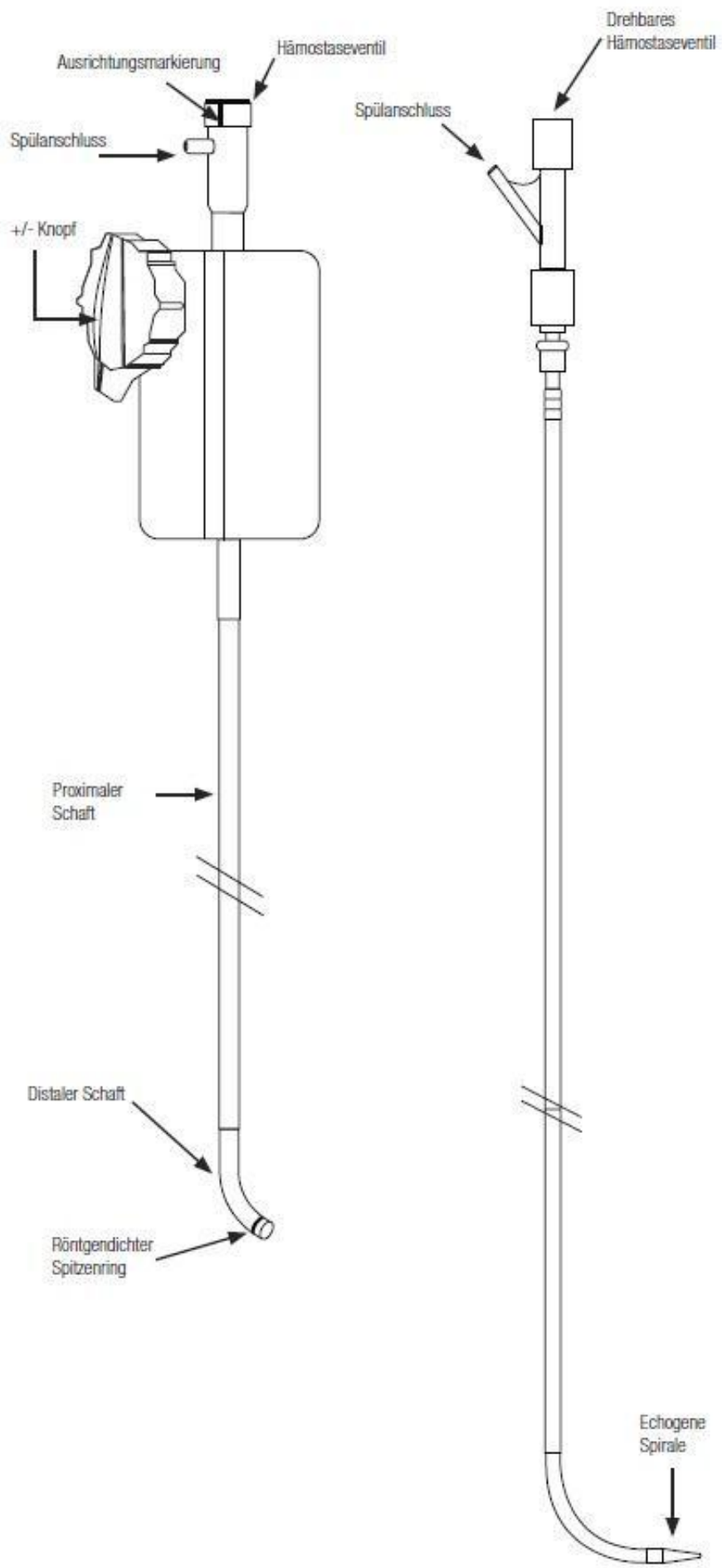

Abb. 2.4: Der steuerbare Führungskatheter (SGC) und der Dilatator (Abbott-Vascular 2008) 


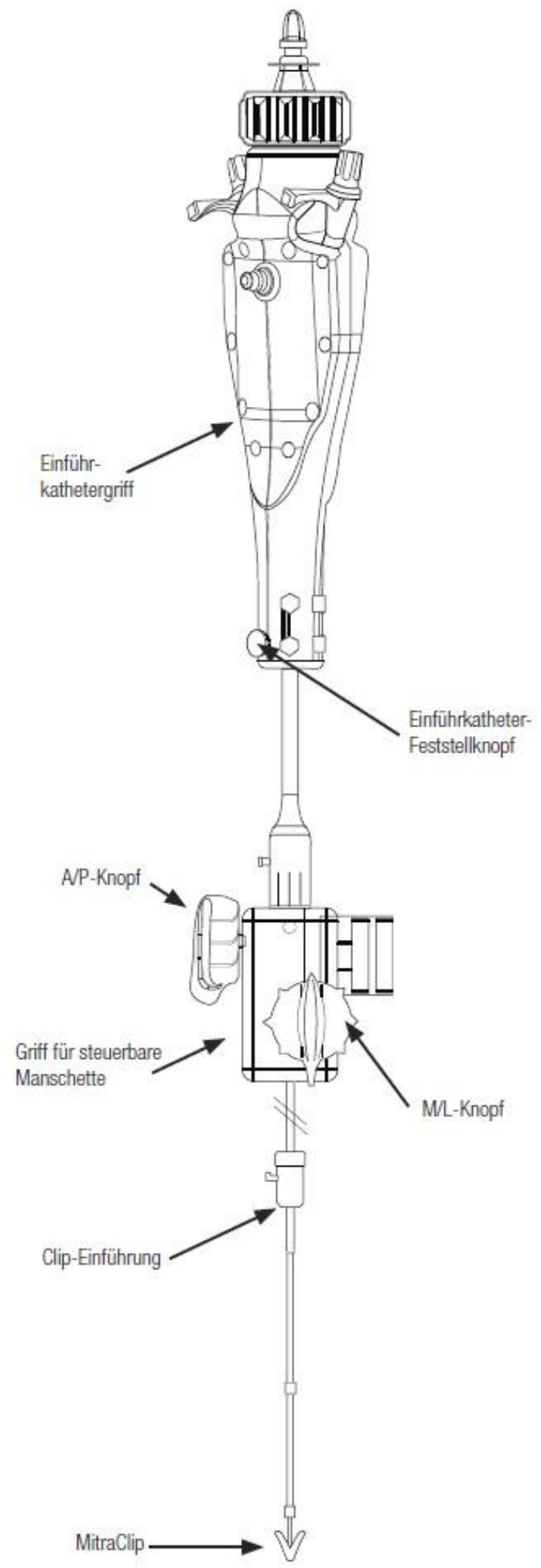

Abb. 2.5: Das Clip-Einführungssystem (CDS)

(Abbott-Vascular 2008) 


\subsection{Ablauf des MitraClip ${ }^{\circledR}$-Eingriffes}

Der Eingriff wurde in früheren Arbeiten ausführlich beschrieben (Feldman et al. 2005; Feldman et al. 2009; Feldman et al. 2010). Es handelt sich um eine kathetergestützte Methode zur perkutanen Mitralklappen-Rekonstruktion am schlagenden Herzen ohne Einsatz der Herz-Lungen-Maschine. Durch Platzierung eines Clips werden das anteriore und das posteriore Mitralsegel über eine Breite von einigen Millimetern permanent fixiert und eine Doppelöffnung geschaffen. Alle MitraClip ${ }^{\circledR}$ Eingriffe werden im Herzkatheterlabor der Universitätsmedizin Göttingen durchgeführt. Bei den Eingriffen sind ein bis zwei interventionelle Kardiologen, ein Interventionsechokardiographeur, ein Kardioanästhesist, ein Mitarbeiter der Firma Abbott Vascular sowie Fachpflegekräfte anwesend. Sämtliche Eingriffe werden unter Vollnarkose durchgeführt. Während des Eingriffs wird eine kontinuierliche transösophageale Echokardiopgraphie durchgeführt, unter deren Sicht sowohl die transseptale Punktion als auch das Einbringen und die Positionierung des MitraClips ${ }^{\circledR}$ stattfinden. Bei Bedarf kann zur weiteren Orientierung die Röntgendurchleuchtung eingesetzt werden.

\subsubsection{Vorbereitung und septale Punktion}

Zuerst wird die rechte V. femoralis punktiert, eine Transseptalschleuse (Fast-Cath 8F SL0, $63 \mathrm{~cm}$, St. Jude Medical) in die Vene eingeführt und diese über einen J-Draht unter fluoroskopischer Sicht bis zur V. cava superior vorgeschoben. Jetzt wird über diese Transseptalschleuse eine Transseptalnadel (BKR1 $71 \mathrm{~cm}$, St. Jude Medical) eingeführt. Die Transseptalschleuse mit eingeführter Punktionsnadel wird in das rechte Atrium zurückgezogen, bis ein „Tenting“, also eine Vorwölbung des Septums der Pars membranacea des atrialen Septums, im transösophagealen Echo sichtbar wird (siehe Abb. 2.6 und 2.7). Die Kontrolle dieses „Tentings“ erfolgt sowohl in der bikavalen Achse bei $80-110^{\circ}$ als auch in der basalen Kurzachse bei 30-60 (vgl. Abb. 2.6 und 2.7). 

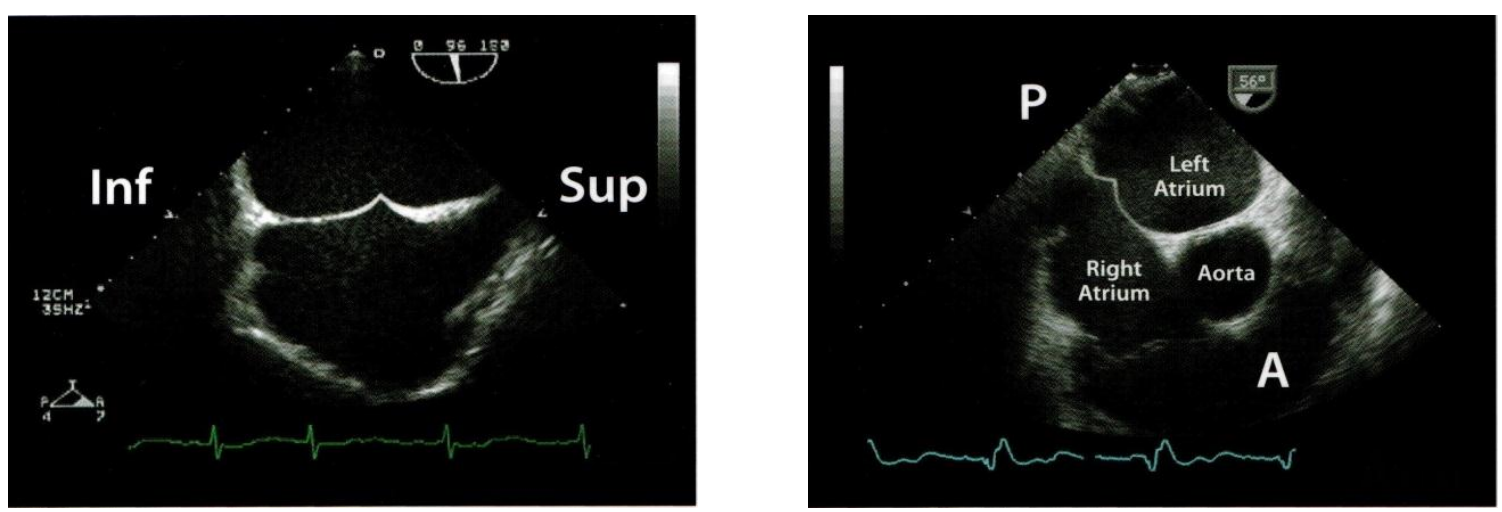

Abb. 2.6 und 2.7: „Tenting“ in der bikavalen Achse 30-60 (links) und in der basalen Kurzachse (rechts). (Abbott-Vascular 2010)

Daraufhin wird der Abstand zwischen dem Punkt der stärksten Vorwölbung und der Koaptationsfläche der Mitralklappe in der Klappenebene gemessen. Die Abstandsmessung erfolgt im 4-Kammerblick bei $0^{\circ}$ (vgl. Abb. 2.8). Beträgt der Abstand $40-45 \mathrm{~mm}$ wird das Vorhofseptum in posteriorer Lage punktiert und die Transseptalschleuse in das linke Atrium vorgeschoben.

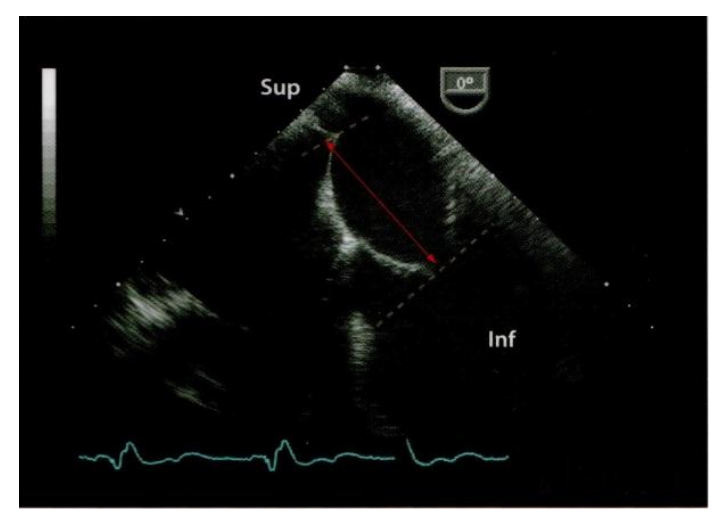

Abb. 2.8: 4-Kammerblick $0^{\circ}$. (Abbott-Vascular 2010)

Nach erfolgreicher Punktion wird die ACT (activated clotting time) durch fraktionierte Gabe von Heparin auf 250 - 300 Sekunden eingestellt. Die ACT wird im Verlauf wiederholt kontrolliert. 


\subsubsection{Positionierung des MitraClip ${ }^{\circledR}$-Systems}

Nach Entfernung der Transseptalschleuse wird der Führungskatheter über den Führungsdraht (Amplatz extra stiff 35/260, Boston Scientific) in das linke Atrium eingebracht, wobei der Dilatator am Ende des Führungskatheters die Punktionsstelle des atrialen Septums ausreichend dilatiert. Nach Einbringen des Führungskatheters wird sowohl der Führungsdraht als auch der Dilatator zurückgezogen, damit im nächsten Schritt das Clip-Einführungssystem (CDS) eingeführt werden kann. Nach Vorschieben des Clip-Einführungssystems über den Führungskatheter in das linke Atrium erfolgt die Positionierung des Clips über der Mitralklappe. Dies geschieht mittels der Handgriffe am Clip-Einführungssystem und wird in mehreren Echokardiographieansichten kontrolliert. Abb. 2.9 zeigt die interkommissurale Achse im 2-Kammerblick bei 55-75 , in welcher die Ausrichtung sowohl nach medial als auch nach lateral kontrolliert wird. Abb. 2.10 zeigt den Punkt des maximalen Insuffizienzjets in der interkommissuralen Achse, über dem der Clip positioniert werden soll.
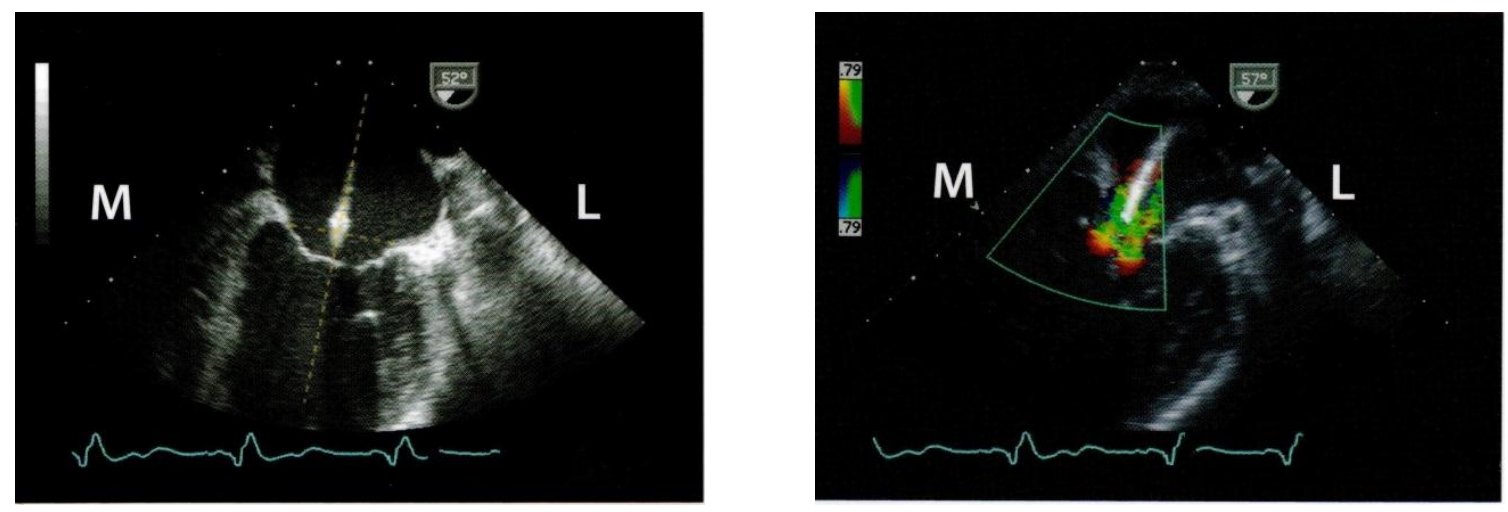

Abb. 2.9 und 2.10: Interkommissurale Achse $55-75^{\circ}$ (links) und Interkommissurale Achse (rechts). (Abbott-Vascular 2010)

Abb. 2.11 und 2.12 zeigt die Ausrichtung sowohl nach anterior als auch nach posterior in der LVOTEinstellung bei $100-160^{\circ}$. 

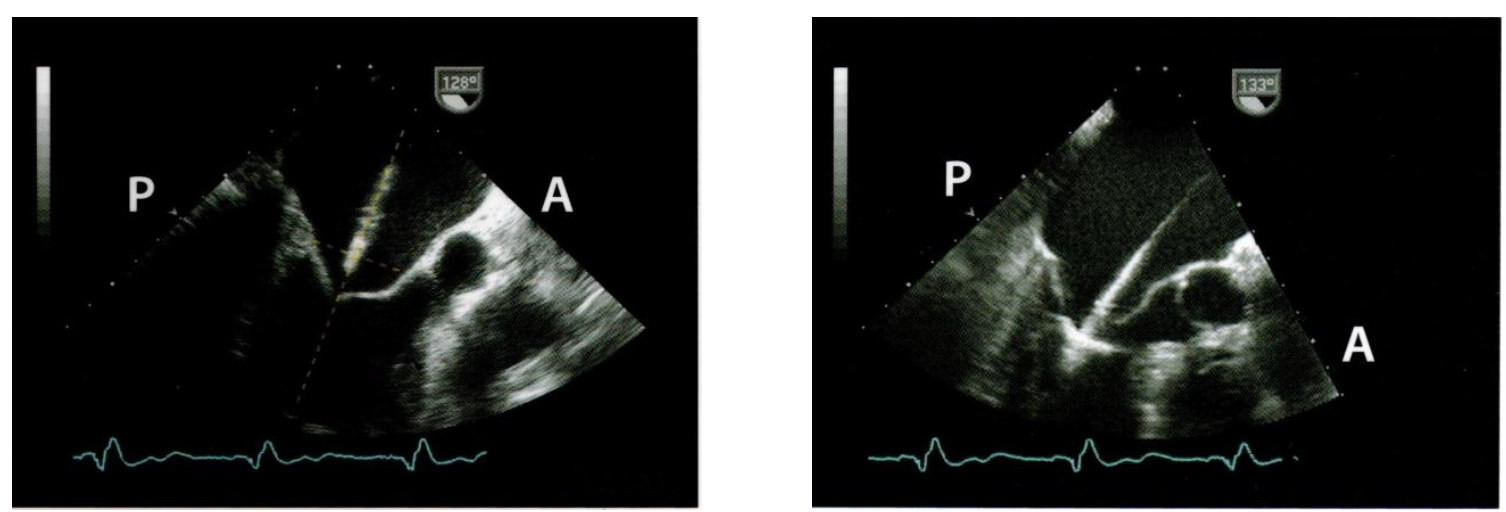

Abb. 2.11 und 2.12: LVOT 100-160 (links) und LVOT 180 (rechts). (Abbott-Vascular 2010)

Abb. 2.13 zeigt, wie der Clip perpendikulär zur Koaptationslinie ausgerichtet wird. Dies geschieht unter echokardiographischer Kontrolle in der Mitralklappenaufsicht bei $0-30^{\circ}$ in der transgastrischen Kurzachse.

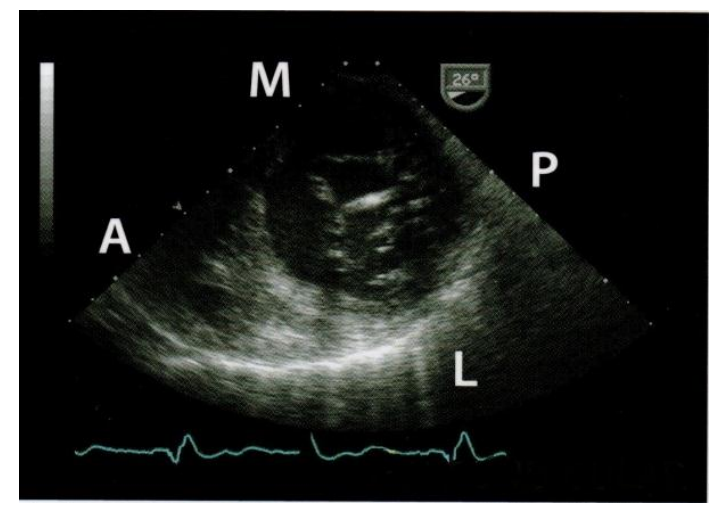

Abb. 2.13: Transgastrische Kurzachse $0-30^{\circ}$. (Abbott-Vascular 2010)

\subsubsection{Platzierung des MitraClips ${ }^{\circledR}$}

Nach Kontrolle der Position des Clips in den oben genannten echokardiographischen Ansichten und optimaler Platzierung des Clips über dem Ursprung des maximalen Insuffizienzjets wird der Clip zwischen den Mitralklappensegeln hindurch in den linken Ventrikel vorgeschoben. Daraufhin werden die Greifarme des Clips geöffnet, so dass sich die beiden Mitralklappensegel beim Zurückziehen des Systems auf die jeweiligen Greifarme auflegen. Durch kleine Widerhaken auf den Greifarmen wird ein mögliches Verrutschen der Segel auf den Greifarmen verhindert. Nach 
erfolgreichem Aufladen der Segel kann der Clip geschlossen werden, was zur Annäherung der beiden Segel führt. Nun wird echokardiographisch überprüft, ob der Clip optimal positioniert ist, ob es zur Bildung eines „double-orifice" gekommen ist und ob eine Reduktion der Mitralinsuffizienz stattgefunden hat. Dies wird unter anderem in der transgastrischen Kurzachse kontrolliert (vgl. Abb. 2.14).

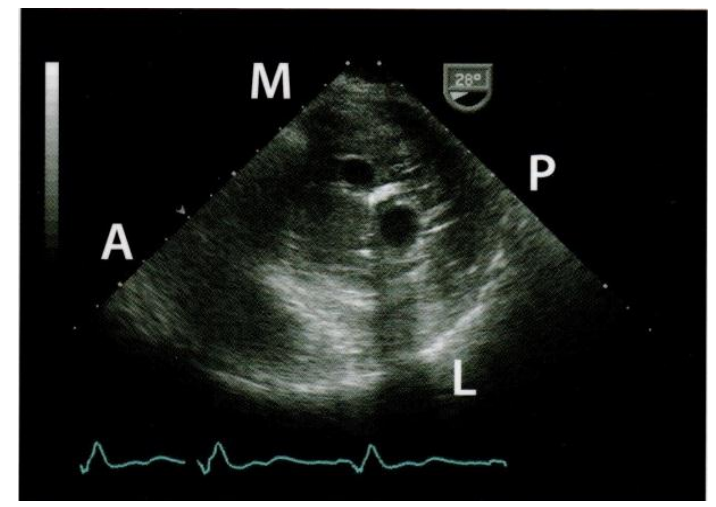

Abb. 2.14: Transgastrische Kurzachse $0-30^{\circ}$. (Abbott-Vascular 2010)

Wurde der Clip nicht optimal positioniert oder keine ausreichende Reduktion der Mitralinsuffizienz erreicht, kann der Clip jederzeit wieder geöffnet werden und nach erneuter Positionierung ein weiterer Clipversuch gestartet werden.

Sind die Position des Clips und die Reduktion der Mitralinsuffizienz erfolgreich, so wird überprüft, ob es zu einer eventuellen Mitralklappenstenose gekommen ist. Wenn der Druckgradient über der Mitralklappe geringer als $3 \mathrm{mmHg}$ ist, wird der Clip vom Einführungssystem gelöst. Ist noch immer eine signifikante Mitralinsuffizienz vorhanden, können weitere Clips platziert werden, bis eine ausreichende Reduktion erreicht ist. Nach Erreichen eines zufriedenstellenden Ergebnisses werden Clip-Einführungssystem und Führungskatheter entfernt und die Punktionsstelle an der rechten V. femoralis mit einer Z-Naht verschlossen. 


\subsection{Echokardiographie}

Sowohl für die transösophageale Echokardiographie (TEE) während des MitraClip ${ }^{\circledR}$-Eingriffes als auch bei der transthorakalen Echokardiographie bei den Kontrolluntersuchungen vor dem Eingriff, nach 6 Monaten und nach 12 Monaten wurde bei allen 36 Patienten das Echokardiographiegerät „Sono-7500" des Herstellers Philips mit verschiedenen Schallköpfen verwendet. Qualitative und quantitative Echokardiographie und die Quantifizierung der Mitralinsuffizienz wurden nach den Leitlinien der American Society of Echocardiography von einem erfahrenen Kardiologen vorgenommen (Zoghbi et al. 2003). Linksventrikuläre Volumina und Ejektionsfraktionen wurden nach der biplanen Methode nach Simpson bestimmt (Lang et al. 2005).

\subsection{Logistischer EuroSCORE}

Der logistische EuroSCORE (European System for Cardiac Operative Risk Evaluation) stellt ein recht einfach durchzuführendes und objektives Verfahren zur Abschätzung der postoperativen 30-TageMortalität herzchirurgischer Patienten dar (siehe Anhang 2). In einer großen europäischen Studie (128 Kliniken, 8 europäische Länder) wurden 17 Risikofaktoren auf der Grundlage von 19.030 Patientendaten identifiziert, welche einen signifikanten Einfluss auf die 30-Tage-Mortalität nach einem herzchirurgischen Eingriff haben (Roques et al. 1999). Auf Basis dieser Ergebnisse und der weiteren Auswertung der 13.302 Patienten aus der EuroSCORE-Datenbank wurde der sogenannte logistische EuroSCORE entwickelt, der eine genauere Berechnung des Risikos eines Patienten erlaubt, innerhalb von 30 Tagen nach einem herzchirurgischen Eingriff zu versterben (Nashef et al. 1999). Für jeden Risikofaktor eines Patienten werden dabei Punkte vergeben. Der logistische EuroSCORE ergibt dabei die geschätzte 30-Tage-Mortalität in Prozent (Roques et al. 2003). 


\subsection{STS-Score}

Der STS-Score (Society of Thoracic Surgeons) ist ebenfalls ein Verfahren zur Abschätzung der operativen 30-Tage Mortalität in der Herzchirurgie. Der Mortalitätsrechner unterscheidet verschiedene Eingriffe, darunter vor allem die Bypasschirurgie und die Herzklappenchirurgie. Neben der Mortalität können außerdem andere Risikowahrscheinlichkeiten berechnet werden, wie z.B. das Risiko eines Schlaganfalles oder eines Nierenversagens. Für den STS-Score werden deutlich mehr Informationen benötigt als für den logistischen EuroSCORE. In dieser Arbeit wird ausschließlich die errechnete Mortalität beobachtet. Für die Berechnung wurde der OnlineCalculator der Society of Thoracic Surgeons eingesetzt (www.sts.org).

\subsection{NT-proBNP}

Das NT-proBNP (N-terminal prohormone of brain natriuretic peptide) ist ein Herzinsuffizienzmarker, welcher sowohl für das Screening als auch zur Diagnosestellung der Herzinsuffizienz benutzt wird und dessen Plasmaspiegel im Verlauf für diese Arbeit bestimmt wurde. Hohe Plasma-NT-proBNP-Spiegel sind prognostisch ungünstig und bei Patienten mit einem schlechten Outcome höher (Bhalla et al. 2004). Die Plasmaspiegel des NT-proBNP sind bei höhergradiger Mitralinsuffizienz erhöht und korrelieren positiv mit der NYHA-Klasse (Yusoff et al. 2006). Sowohl LVEDV als auch LVESV korrelieren signifikant positiv mit der Höhe des Plasma-NTproBNP. Des Weiteren steigt das NT-proBNP in zunehmendem Maße mit einer Verschlechterung der Herzinsuffizienzsymptome und steht in Verbindung mit dem Ausmaß des linksventrikulären Remodeling (Yusoff et al. 2006). 


\subsection{Standardisierter 6-Minuten-Gehtest}

Der standardisierte 6-Minuten-Gehtest ist ein Belastungstest, bei dem die Patienten unter standardisierten Bedingungen aufgefordert werden, innerhalb von 6 Minuten so weit wie möglich zu gehen und somit eine möglichst große Strecke zurück zu legen. In der vorliegenden Studie wurde die erreichte Gehstrecke als Faktor für Leistungsfähigkeit im Verlauf ausgewertet. Standardisiert wird dieser Test dadurch, dass der Untersucher die während des Tests zurückgelegte Gehstrecke anhand von Markierungen auf dem Fußboden in Metern dokumentiert und die Teststrecke bei jedem Test dieselbe ist. Des Weiteren wurde der Test immer unter den gleichen Bedingungen mit den gleichen Kommandos und Instruktionen durchgeführt. Eine Studie zeigte, dass eine Verlängerung der Gehstrecke um 70 Meter oder mehr von den Patienten als klinisch relevant wahrgenommen wurde (Redelmeier et al. 1997).

\subsection{Minnesota Living With Heart Failure Questionnaire (MLHFQ)}

Der MLHFQ ist ein Fragebogen zur Bewertung des therapeutischen Effektes von Eingriffen bei Herzinsuffizienz, bei welchem sich die Patienten selbst beurteilen sollen. Er beinhaltet 21 Fragen und wurde an der Universität von Minnesota, Minneapolis USA entwickelt (Rector und Cohn 1992). Er misst die subjektive Wahrnehmung der Auswirkungen chronischer Herzinsuffizienz auf das Leben der Patienten in den letzten 4 Wochen. Diese Auswirkung wird für jede spezifische Einschränkung hinsichtlich der Stärke der Behinderung (von $0=$ Nein bis $5=I m m e r$ ) einstuft. Die Summe aller Einzelantworten bilden den Summenscore, d.h. maximal 105 Punkte (105 Punkte $=$ schwere Behinderung, 0 Punkte $=$ keine Behinderung). Es lässt sich ebenfalls ein Wert für die körperliche Dimension als auch für die emotionale Dimension berechnen (Burkart et al. 2006; Quittan et al. 2001), was in dieser Arbeit jedoch nicht durchgeführt wurde. In dieser Arbeit wurde der gesamte Summenscore als subjektives Korrelat des Erfolges ausgewertet, es wurden keine Untergruppen gebildet. 


\subsection{Statistische Auswertung}

Die Analyse der Daten und deren statistische Auswertung erfolgte mit Unterstützung der Abteilung Medizinische Statistik der Universitätsmedizin Göttingen. Die Auflistung und Auswertung aller gesammelten Daten, die Berechnung von Medianen, Quartilen, Mittelwerten und Konfidenzintervallen sowie die graphische Darstellung der erfassten Parameter und aller abzubildenden Daten wurden mit Hilfe von Microsoft Excel 2010 durchgeführt. Normal verteilte Variablen sind als Mittelwert $\pm 95 \%$ Konfidenzintervall, nicht normal verteilte Variablen als Median (25 - 75\% Quartil) dargestellt.

Analysen wurden mit „Statistica 9.1“ (StatSoft, Inc.) und „SAS 9.2“ (SAS-Institute, Inc) durchgeführt. Um signifikante Unterschiede über die Zeit festzustellen, wurde für normalverteilte Werte eine ANOVA für Messwiederholungen durchgeführt. Nichtnormalverteilte Werte wurden zunächst geranked und dann ebenfalls mit einer ANOVA für Messwiederholungen auf Signifikanz getestet.

Es wurde ein $p<0,05$ als statistisch signifikant, ein $p<0,01$ als hochsignifikant und ein $p<0,001$ als höchstsignifikant angenommen.

Nachdem die Globalhypothese zum 5 \% Niveau abgelehnt wurde, wurde ein gepaarter t-Test für abhängige Stichproben durchgeführt. In diesem Test wurde das Signifikanzniveau nach Bonferroni adjustiert. Als neues Signifikanzniveau ergibt sich 2,5\%, also wird ein $p<0,025$ als signifikant, ein $p<0,005$ als hochsignifikant und ein $p<0,00025$ als höchstsignifikant gewertet.

Die Überlebenswahrscheinlichkeiten wurden nach Kaplan-Meier analysiert. 


\section{Ergebnisse}

\subsection{Patientencharakteristika}

Tab. 3.1 zeigt Basis-Charakteristika der 36 Patienten. Alle Patienten litten an einer signifikanten Mitralinsuffizienz (Median Grad 4, Tab. 3.1), davon 72,2\% an einer funktionellen, 27,8\% an einer organischen Mitralinsuffizienz. Alle Patienten zeigten eine deutliche Symptomatik und waren überwiegend den Herzinsuffizienz-Klassen NYHA 3 und 4 zugeordnet. Die 6-Minuten-Gehstrecke war mit $228 \pm 55$ m erheblich eingeschränkt (vgl. auch Tab. 3.1). Es handelte sich um Patienten im mittleren Alter von $73 \pm 3$ Jahren mit erhöhtem operativem Risiko bei einer berechneten operativen Mortalität von $32 \pm 5 \%$ (logistischer EuroScore) bzw. $12 \pm 3 \%$ (STS-Score). Das Risiko der Patienten wurde durch einen hohen Anteil an relevanten Begleiterkrankungen determiniert (vgl. Tab. 3.1). Aufgrund von Kontraindikationen wären nur $19,4 \%$ der Patienten in die multizentrische, randomisierte EVEREST-II-Studie (Feldman et al. 2010) eingeschlossen worden.

\begin{tabular}{|l|c|}
\hline \multicolumn{2}{|c|}{ Basis-Charakteristika } \\
\hline \multicolumn{2}{|c|}{} \\
\hline MW \pm 95\% K.I. \\
\hline Alter, Jahre \\
\hline Berechnete OP-Mortalität \\
\hline Log. EuroScore, \% \\
\hline STS Score, \% & $32 \pm 6$ \\
\hline LV-EF, \% & $12 \pm 3$ \\
\hline 6-Minuten-Gehtest, m & $35 \pm 5$ \\
\hline Median (Min - Max) & $228 \pm 55$ \\
\hline Grad der Mitralinsuffizienz \\
\hline NYHA-Klasse & $4(3-4)$ \\
\hline n (\%) & $3(2-4)$ \\
\hline Weibliches Geschlecht & $10(27,8 \%)$ \\
\hline Ätiologie der MI & \\
$-\quad$ organisch & $10(27,8 \%)$ \\
\hline funktionell & $26(72,2 \%)$ \\
\hline LV-EF $30 \%$ & $19(52,8 \%)$ \\
\hline CRTD & $13(36,1 \%)$ \\
\hline KHK & $19(52,8 \%)$ \\
\hline Frühere Herzoperation & $16(44,4 \%)$ \\
\hline Pulmonaler Hypertonus & $26(72,2 \%)$ \\
\hline
\end{tabular}




\begin{tabular}{|l|c|}
\hline Vorhofflimmern & $22(61,1 \%)$ \\
\hline pAVK & $10(27,8 \%)$ \\
\hline Früherer Schlaganfall & $1(2,8 \%)$ \\
\hline $\begin{array}{c}\text { Niereninsuffizienz } \\
\quad \text { GFR }^{\#}<30 \mathrm{ml} / \mathrm{min} / 1,73 \mathrm{~m}^{2}\end{array}$ \\
$-\quad$ GFR $^{\#} 30-59 \mathrm{ml} / \mathrm{min} / 1,73 \mathrm{~m}^{2}$ & $9(25 \%)$ \\
\hline COPD & $18(50 \%)$ \\
\hline Diabetes mellitus & $9(25 \%)$ \\
\hline Positiv inotrope Substanzen & $12(33,3 \%)$ \\
\hline Eignung für EVEREST II & $5(13,9 \%)$ \\
\hline \multicolumn{2}{|c|}{ \# berechnet mit der MDRD-Formel (Levey et al. 1999) } \\
\hline \multicolumn{2}{|c|}{}
\end{tabular}

Tab. 3.1: Patientencharakteristika

\subsection{Follow-Up-Qualität}

Alle überlebenden Patienten wurden nach $6 \pm 2$ Monaten und nach $12 \pm 2$ Monaten zum Follow-Up einbestellt. Für das Erstellen der Kaplan-Meier-Kurve wurden zudem noch einmal alle Patienten angerufen und nach eventuellen Komplikationen wie zum Beispiel Hospitalisationen aufgrund von kardialer Dekompensation gefragt. Bei den Anrufen betrug das mediane Follow-Up 13 (11 - 18) Monate (25 - 75\% Quartil). Bei dem regulären Follow-Up Termin nach 6 Monaten sind 4 Patienten vor Erreichen der 6-Monats-Kontrolle verstorben und 2 Patienten nicht zum Termin erschienen. 6Monats-Follow-Up-Daten liegen von 30 der 32 überlebenden Patienten (93,7\%) vor. Beim regulären Follow-Up Temin nach 12 Monaten sind insgesamt 7 der ursprünglich 36 Patienten vor Erreichen der 12-Monats-Kontrolle verstorben und wiederum 2 Patienten nicht zum Termin erschienen. Somit lagen 12-Monats-Follow-Up-Daten von 27 der 29 überlebenden Patienten (93,1\%) vor.

Die Analysen der im Follow-Up bestimmten Werte über 12 Monate wurden an den überlebenden Patienten durchgeführt. Von $n=27$ abweichende Patientenzahlen in diesen Abbildungen sind durch fehlende Werte einzelner Patienten zu erklären. 


\subsection{Prozedurale Daten}

Der Eingriff war bei 35 Patienten (97,2\%) erfolgreich. Ein Eingriff wurde als erfolgreich gewertet, wenn mindestens 1 MitraClip ${ }^{\circledR}$ implantiert wurde und nach Ermessen des Katheteriseurs ein funktionell zufriedenstellendes Ergebnis erzielt wurde. Die mediane Gesamteingriffsdauer (SchnittNaht-Zeit) lag bei 150 min, die mediane Dauer von der Einführung bis zur Entfernung des Führungskatheters (Systemzeit) bei 98 min. 1 Patient (2,8\%) hat 0 Clips erhalten, 19 (52,8\%) 1 Clip, 14 (38,9\%) 2 Clips und 2 Patienten (5,6\%) haben 3 Clips erhalten. Weitere prozedurale Daten sind in Tab. 3.2 aufgelistet.

\begin{tabular}{|l|c|}
\hline \multicolumn{2}{|c|}{ Prozedurale Daten } \\
\hline $\mathrm{n}(\%)$ & $35(97,2 \%)$ \\
\hline Prozedurerfolg & $150(114-225)$ \\
\hline Median (25-75\%) & $98(66-149)$ \\
\hline Schnitt-Naht-Zeit, min & $26(18-42)$ \\
\hline System-Zeit ${ }^{\prime}$ min & $1(1-2)$ \\
\hline Durchleuchtungszeit, min & \\
\hline Anzahl Clips, $\mathrm{n}$ & \\
\hline \multicolumn{2}{|c|}{${ }^{\text {\# } \text { Zeit von der Einführung bis zur Entfernung des Führungskatheters }}$} \\
\hline
\end{tabular}

Tab. 3.2: Prozedurale Daten 


\subsection{Effektivität des Eingriffs}

Die Prozedur konnte bei 35 Patienten (97,2\%) erfolgreich durchgeführt werden. Bei 30 Patienten (83,3\%) wurde eine Reduktion der Mitralinsuffizienz auf einen Grad $\leq 2$ erzielt (Tab. 3.3). Dies entspricht einer medianen Reduktion um 2 Grade.

\subsection{Eingriffssicherheit (30-Tage-Ergebnisse)}

Der Eingriff erwies sich als sicher. Es gab einen Todesfall aufgrund von refraktärer Sepsis bei Pneumonie innerhalb der ersten 30 Tage nach Eingriff. Dies entspricht einer 30-Tage-Mortalität von 2,8\%, was einer berechneten durchschnittlichen operativen Mortalität von 32\% (EuroScore) bzw. 12\% STS-Score gegenübersteht. 6 Patienten $(16,7 \%)$ wurden innerhalb von 30 Tagen wegen dekompensierter Herzinsuffizienz erneut hospitalisiert. Darunter waren 5 Patienten mit schwer reduzierter LV-Funktion (LV-EF $\leq 30 \%$ ) vor Clip-Implantation. Kein Patient mit früher ReHospitalisierung hatte ein funktionell insuffizientes Ergebnis an der Mitralklappe mit einer residualen Mitralinsuffizienz Grad 3 oder 4. Bei 3 Patienten (8,3\%) traten schwere Blutungen (GUSTO (GUSTO-Investigators 1993)) mit hämodynamischer Instabilität auf. Es traten 3 Fälle (8,3\%) mit partieller Cliplösung von einem Segel auf. Vollständige Cliplösungen und -embolisationen sind dagegen nicht aufgetreten. Weitere Ereignisse sind in Tab. 3.3 aufgelistet. Es traten keine Fälle von Myokardinfarkt, Schlaganfall, Perikardtamponade oder Notwendigkeit von Herz- oder Gefäßoperation oder mechanischer Kreislaufunterstützung auf. 


\begin{tabular}{|l|c|}
\hline \multicolumn{2}{|c|}{ 30-Tage-Ergebnisse } \\
\hline \multicolumn{2}{|c|}{$n(\%)$} \\
\hline Effektivität & $30(83,3 \%)$ \\
\hline Mitralinsuffizienz Grad $\leq 2$ & $1(2,8 \%)$ \\
\hline Sicherheit & $6(16,7 \%)$ \\
\hline Tod & $3(8,3 \%)$ \\
\hline Hospitalisierung wegen Herzinsuffizienz & $0(0 \%)$ \\
\hline Schwere Blutung (GUSTO) & $0(0 \%)$ \\
\hline Myokardinfarkt & $0(0 \%)$ \\
\hline Schlaganfall & $0(0 \%)$ \\
\hline Herzoperation & $0(0 \%)$ \\
\hline Mechanische Kreislaufunterstützung & $4(11,1 \%)$ \\
\hline Perikardtamponade & $0(0 \%)$ \\
\hline Beatmung $>$ 24h & $1(2,8 \%)$ \\
\hline Phlebothrombose & $3(8,3 \%)$ \\
\hline Akutes Nierenversagen & $0(0 \%)$ \\
\hline $\begin{array}{l}\text { Cliplösung: } \\
\text { partiell } \\
\text { komplett }\end{array}$ & \\
\hline $\begin{array}{c}\text { \# Per definitionem: Intrakranielle Blutung oder Blutung mit interventionsbedürftiger } \\
\text { hämodynamischer Auswirkung (GUSTO (GUSTO-Investigators 1993)); im vorliegenden Kollektiv } \\
\text { sind keine intrakraniellen Blutungen aufgetreten }\end{array}$ \\
\hline
\end{tabular}

Tab. 3.3: 30-Tage-Ergebnisse

\subsection{Korrektureingriffe}

Bei 4 Patienten (11,1\%) wurden 79, 81, 287 bzw. 301 Tage nach Clip Korrektureingriffe aufgrund eines Rezidivs der Mitralinsuffizienz durchgeführt. 1 Patient wurde einem zweiten Clipeingriff unterzogen. Bei diesem Patienten konnte mit dem Zweiteingriff eine deutliche Reduktion der Mitralinsuffizienz auf Grad 1 erzielt werden. 3 Patienten wurden einem operativen Mitralklappenersatz unterzogen. 


\subsection{Beeinflussung des Mitralinsuffizienz-Grades über 12 Monate}

Abb. 3.1 zeigt den Verlauf des Mitralinsuffizienz-Grades über den Studienzeitraum. Nach 12 Monaten zeigten 76,9\% der überlebenden Patienten eine Mitralinsuffizienz Grad $\leq 2$. Als kontinuierliche Variable betrachtet zeigten sich vor und nach Clip sowie nach 6 und 12 Monaten Mitralinsuffizienzen des Grades 3,6 $\pm 0,2,1,5 \pm 0,2,2,0 \pm 0,4$ und 1,8 $\pm 0,3(p<0,0001)$. Es lassen sich globale hochsignifikante Unterschiede $(p<0,0001)$ beobachten, welche auch im Verlauf persistieren.

Des Weiteren sind im t-Test sowohl das Ergebnis nach 6 Monaten zu „Vor Clip“ ( $p<0,0001)$, als auch das Ergebnis nach 12 Monaten zu „Vor Clip“ ( $p<0,0001)$ höchstsignifikant.

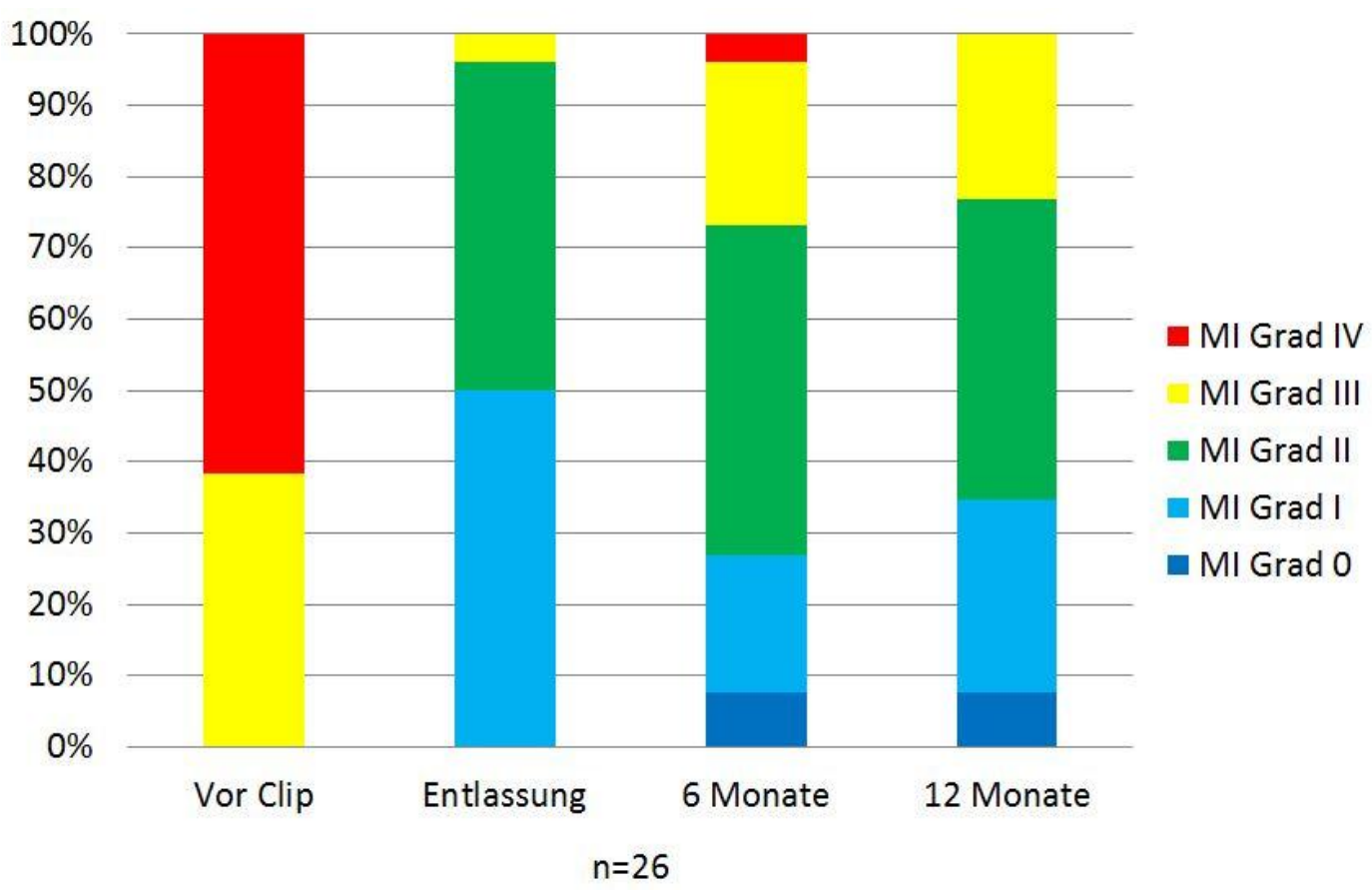

Abb. 3.1: Grad der Mitralinsuffizienz. Höchstsignifikante Abnahme der Schwere der Mitralinsuffizienz nach MitraClip ${ }^{\circledR}$-Implantation und Persistenz des Ergebnisses im Beobachtungszeitraum $(p<0,0001)$. 


\subsection{Der NYHA-Status über 12 Monate}

Abb. 3.2 zeigt den Verlauf der NYHA-Klasse, also der Symptomatik, über den Studienzeitraum. Nach 12 Monaten zeigten 74\% der überlebenden Patienten keine oder geringe Symptome entsprechend NYHA 1 oder 2. Betrachtet man hier NYHA als kontinuierliche Variable vor Clip sowie nach 6 und 12 Monaten, so sieht man folgende Werte: $3,3 \pm 0,2,2,1 \pm 0,3$ und 1,8 8 0,3 ( $p<0,0001)$. Auch hier lassen sich globale hochsignifikante Unterschiede $(p<0,0001)$ beobachten, welche wiederum auch im Verlauf persistieren.

Wie schon bei dem Verlauf der Mitralinsuffizienz zeigten sich auch hier im t-Test sowohl das Ergebnis nach 6 Monaten zu „Vor Clip“ ( $p<0,0001)$, als auch das Ergebnis nach 12 Monaten zu „Vor Clip“ ( $p<0,0001)$ als höchstsignifikant.

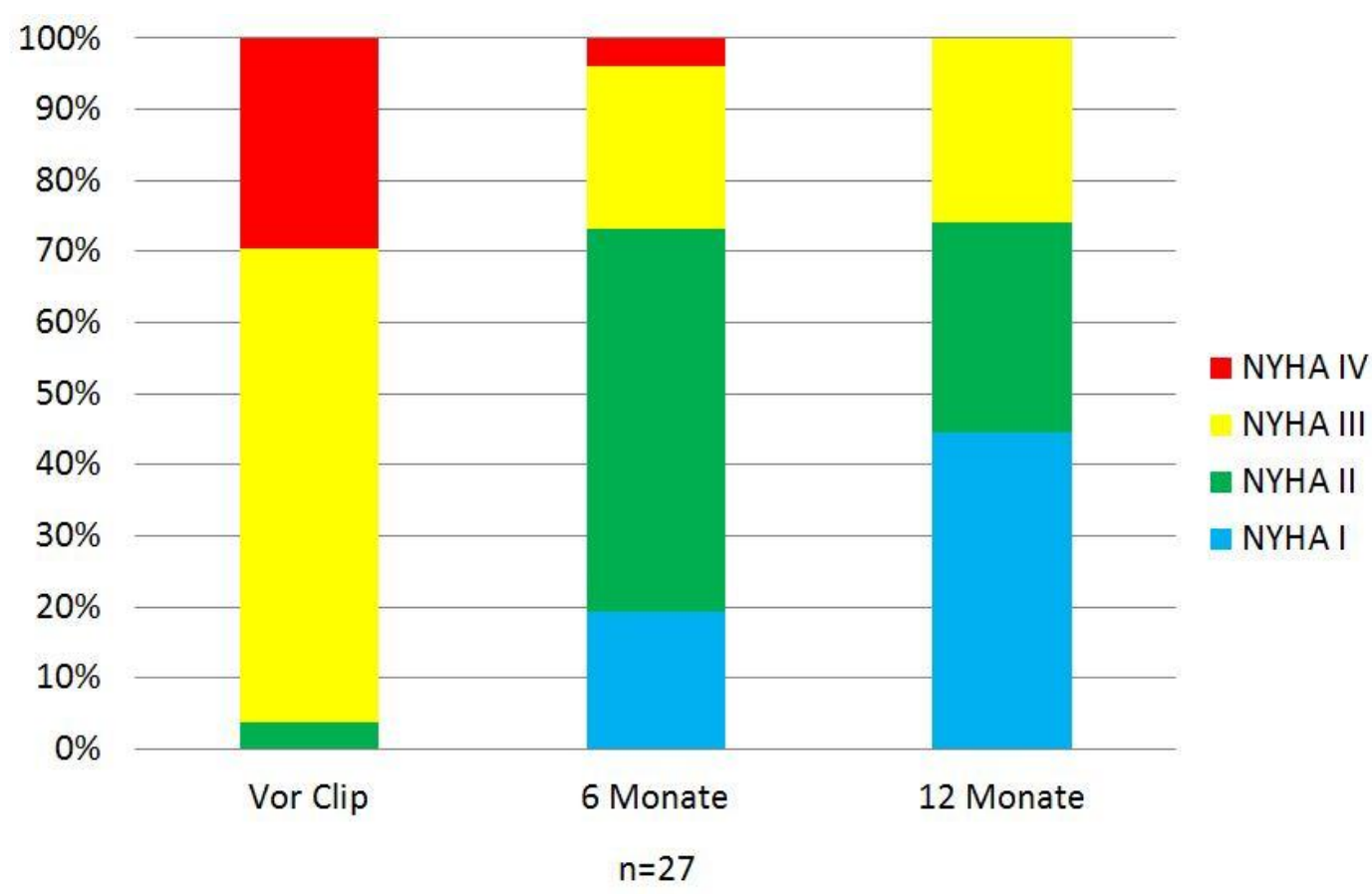

Abb. 3.2: Herzinsuffizienz-Stadium nach NYHA. Höchstsignifikante Besserung der Beschwerdesymptomatik nach NYHA $(p<0,0001)$ nach MitraClip ${ }^{\circledR}$-Implantation über den gesamten Beobachtungszeitraum. 


\subsection{Gehstreckenveränderung über 12 Monate}

Abb. 3.3 zeigt den Verlauf der Gehstrecke im 6-Minuten-Gehtest (6MWT), also der Leistungsfähigkeit sowohl vor, als auch nach dem Eingriff. Es zeigten sich vor Clip, sowie nach 6 und 12 Monaten Gehstrecken von $279 \pm 60,362 \pm 55$ und $375 \pm 54(p<0,001)$. Hier lassen sich globale höchstsignifikante Unterschiede $(p<0,001)$ beobachten, welche auch wiederum im Verlauf persistieren. Es wurde eine durchschnittliche Gehstreckenverlängerung von „Vor Clip“ zu 12 Monaten um 96 m nachgewiesen. Bei dem Verlauf der Gehstrecke zeigten sich die Ergebnisse im tTest sowohl nach 6 Monaten zu „Vor Clip“ $(p<0,01)$, als auch nach 12 Monaten zu „Vor Clip“ ( $p<$ $0,01)$ als signifikant.

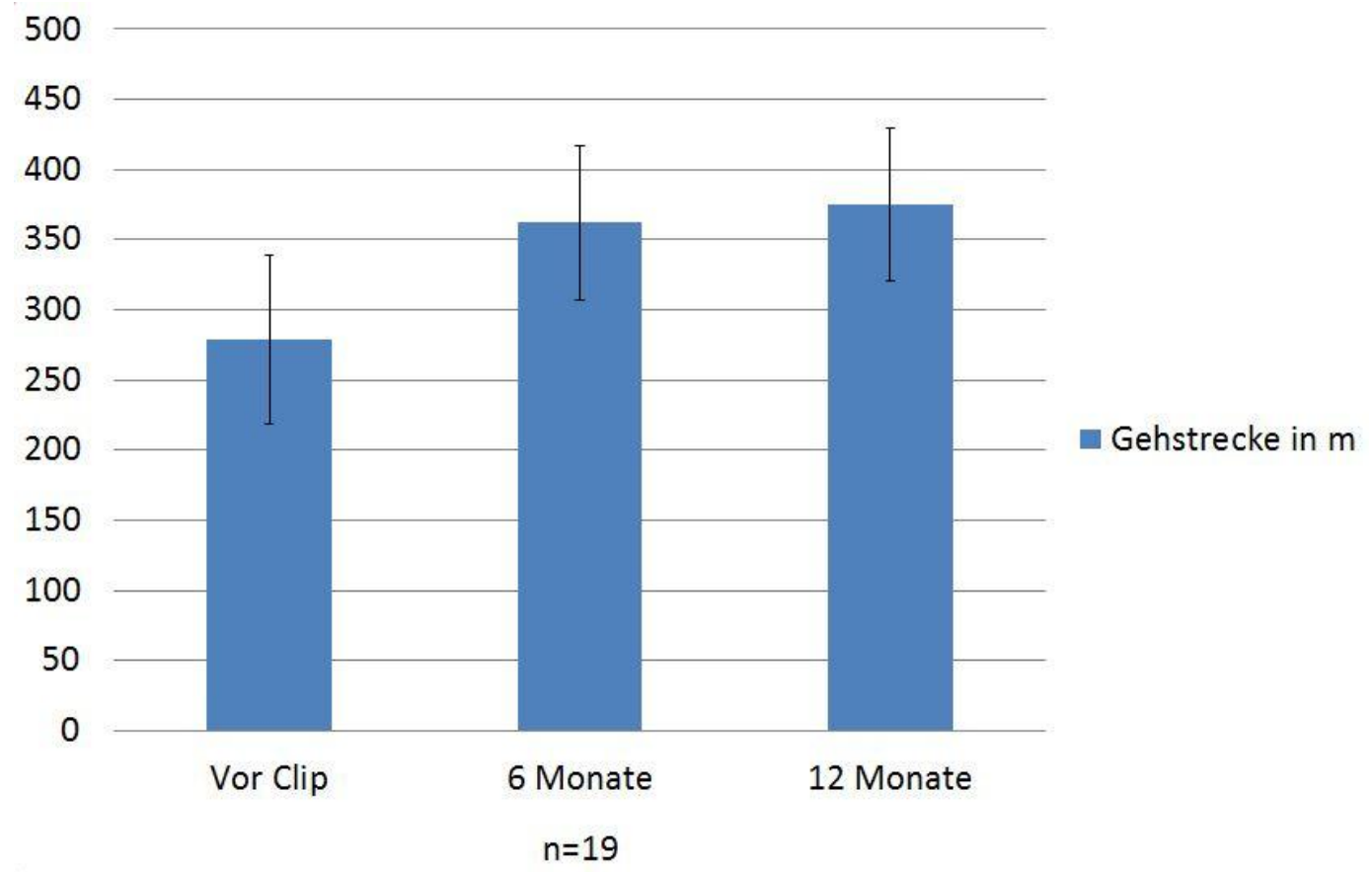

Abb. 3.3: Gehstrecke im 6-Minuten-Gehtest. Höchstsignifikante Besserung der Belastbarkeit $(p<$ 0,001 ) nach MitraClip ${ }^{\circledR}$-Implantation über den gesamten Beobachtungszeitraum. 


\subsection{MLHFQ-Ergebnisse über 12 Monate}

Abb. 3.4 zeigt den MLHFQ-Summenscore (Minnesota Living With Heart Failure Questionnaire) im Verlauf. Es sind Werte zwischen 0 - 105 möglich. Je geringer der Wert, desto besser fühlen sich die Patienten subjektiv. Es zeigten sich vor Clip, sowie nach 6 und 12 Monaten Werte von $47 \pm 9,29 \pm 8$ und $23 \pm 8(p<0,0001)$. Hier lassen sich globale höchstsignifikante Unterschiede $(p<0,0001)$ beobachten, welche auch wiederum im Verlauf persistieren. Es wurde eine durchschnittliche Verminderung von „Vor Clip“ zu 12 Monaten um 24 Punkte nachgewiesen.

Im Verlauf des MLHFQ-Summenscores zeigten sich die Ergebnisse im t-Test sowohl nach 6 Monaten zu „Vor Clip“ ( $p<0,001)$ als auch nach 12 Monaten zu „Vor Clip“ ( $p<0,0001)$ als höchstsignifikant.

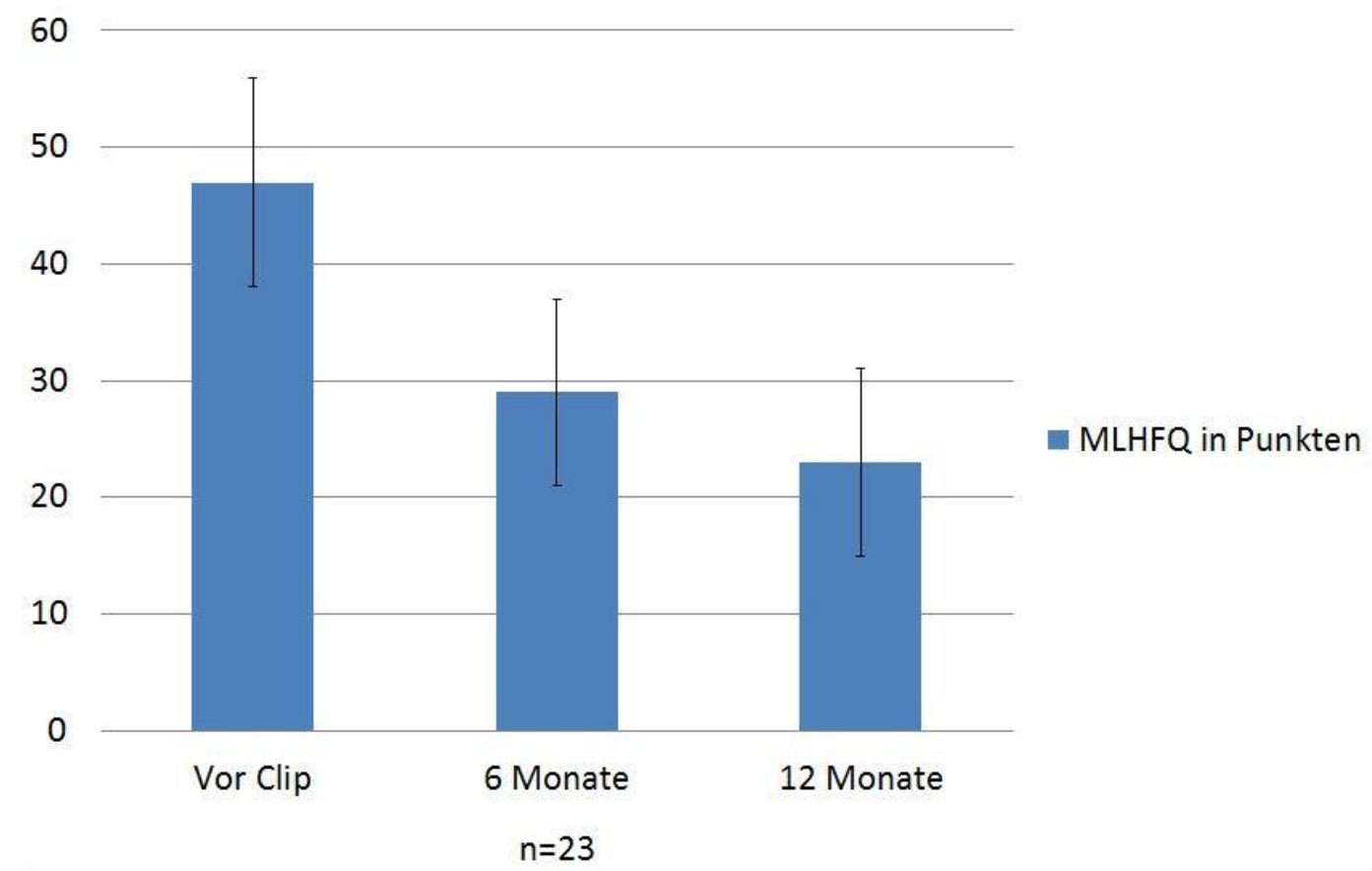

Abb. 3.4: MLHFQ (0 - 105 Punkte) in Punkten. Höchstsignifikante Besserung des subjektiven Befindens $(p<0,001)$ nach MitraClip ${ }^{\circledR}$-Implantation über den gesamten Beobachtungszeitraum. 


\subsection{Reverses Remodeling durch das MitraClip ${ }^{\circledR}$-System}

\subsubsection{Linksventrikuläre Durchmesser}

Abb. 3.5 zeigt sowohl die linksventrikulären enddiastolischen Durchmesser (LVEDD) als auch die linksventrikulären endsystolischen Durchmesser (LVESD) im Verlauf über 12 Monate. Für die LVEDD zeigten sich vor Clip, sowie nach 6 und 12 Monaten Werte von $62 \pm 4,61 \pm 4$ und $58 \pm 4(p<0,001)$. Hier lassen sich globale höchstsignifikante Unterschiede $(p<0,001)$ beobachten. Es wurde eine durchschnittliche Verminderung des LVEDD um $4 \mathrm{~mm}$ gesehen. Bei dem Verlauf der LVEDD zeigten sich die Ergebnisse im t-Test nach 6 Monaten zu „Vor Clip“ als nicht signifikant ( $p=0,03363)$, nach 12 Monaten zu „Vor Clip“ ( $p<0,001)$ jedoch als höchstsignifikant.

Für die LVESD zeigten sich vor Clip, sowie nach 6 und 12 Monaten Werte von 49 \pm 6, $49 \pm 6$ und $47 \pm 6(p=0,27089)$. Hier lassen sich keine globalen höchstsignifikanten Unterschiede $(p=0,27089)$ feststellen.

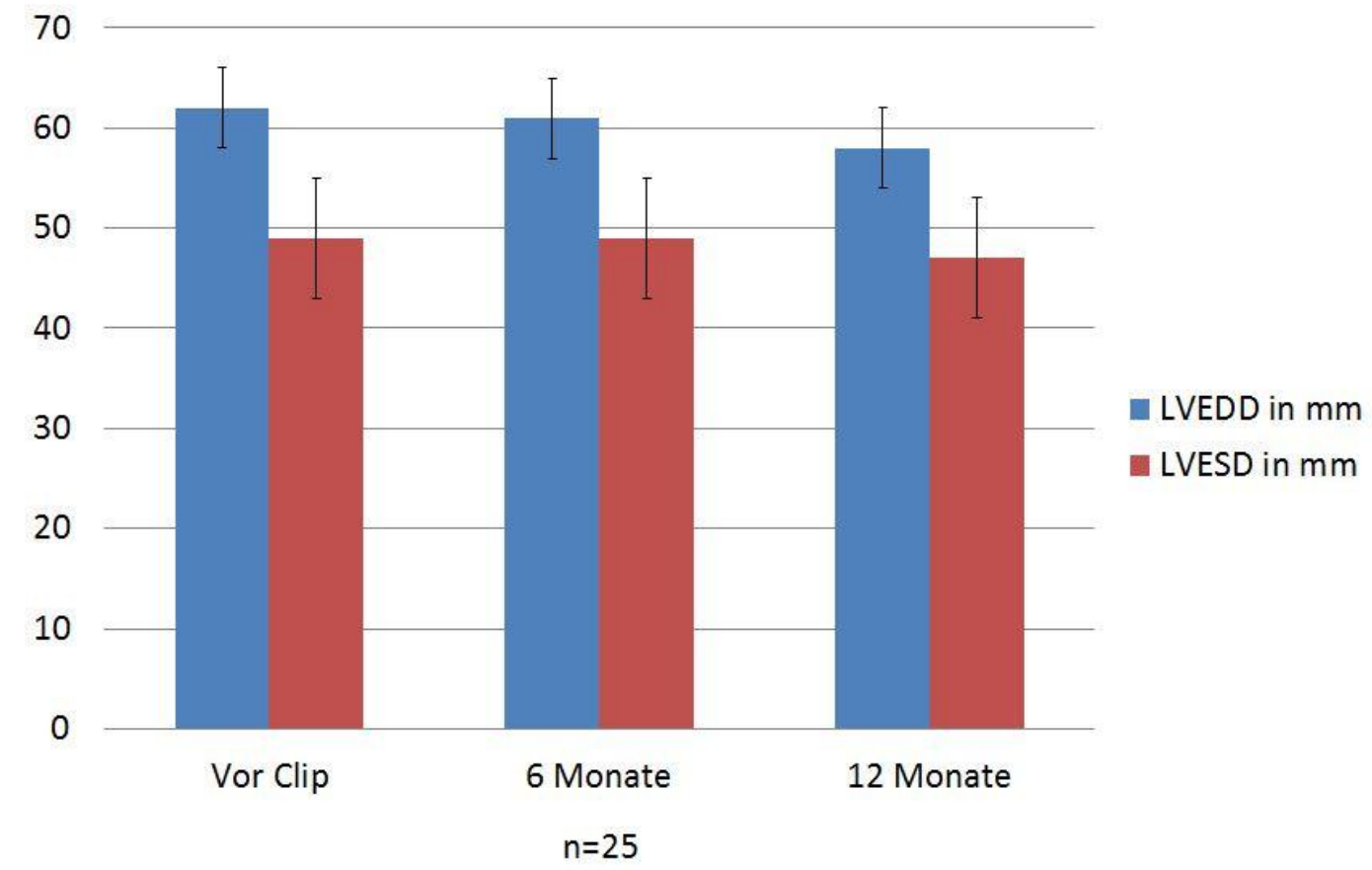

Abb. 3.5: Linksventrikuläre Durchmesser (enddiastolisch und endsystolisch) in $\mathrm{mm}$. Höchstsignifikante Verminderung des $\operatorname{LVEDD}(p<0,001)$, jedoch nicht des $\operatorname{LVESD}(p=0,27089)$ nach MitraClip ${ }^{\circledR}$-Implantation über den gesamten Beobachtungszeitraum. 


\subsubsection{Linksventrikuläre Volumina}

Abb. 3.6 zeigt sowohl die linksventrikulären enddiastolischen Volumina (LVEDV) als auch die linksventrikulären endsystolischen Volumina (LVESV) im Verlauf über 12 Monate. Für die LVEDV zeigten sich vor Clip, sowie nach 6 und 12 Monaten Werte von $214 \pm 44,189 \pm 41$ und $151 \pm 26$ (p< $0,001)$. Hier lassen sich globale höchstsignifikante Unterschiede $(p<0,001)$ beobachten. Es wurde eine durchschnittliche Verminderung des LVEDV von „Vor Clip“ zu 12 Monaten um 63 ml gesehen.

Für die LVESV zeigten sich vor Clip, sowie nach 6 und 12 Monaten Werte von $137 \pm 42,129 \pm 38$ und $97 \pm 24(p<0,05)$. Hier lassen sich globale signifikante Unterschiede $(p<0,05)$ feststellen. Es wurde eine durchschnittliche Verminderung des LVESV von „Vor Clip“ zu 12 Monaten um $40 \mathrm{ml}$ gesehen. Bei dem Verlauf der LVEDV zeigten sich die Ergebnisse im t-Test sowohl nach 6 Monaten zu „Vor Clip“ ( $p<0,01)$, als auch nach 12 Monaten zu „Vor Clip“ $(p<0,01)$ als hochsignifikant.

Beim LVESV zeigten sich die Ergebnisse im t-Test weder nach 6 Monaten zu „Vor Clip“ ( $p=$ 0,361875), noch nach 12 Monaten zu „Vor Clip“ ( $p=0,041825)$ als signifikant. Jedoch zeigten sich die Ergebnisse nach 12 Monaten zu den Ergebnissen nach 6 Monaten im t-Test als signifikant $(p<0,025)$. 


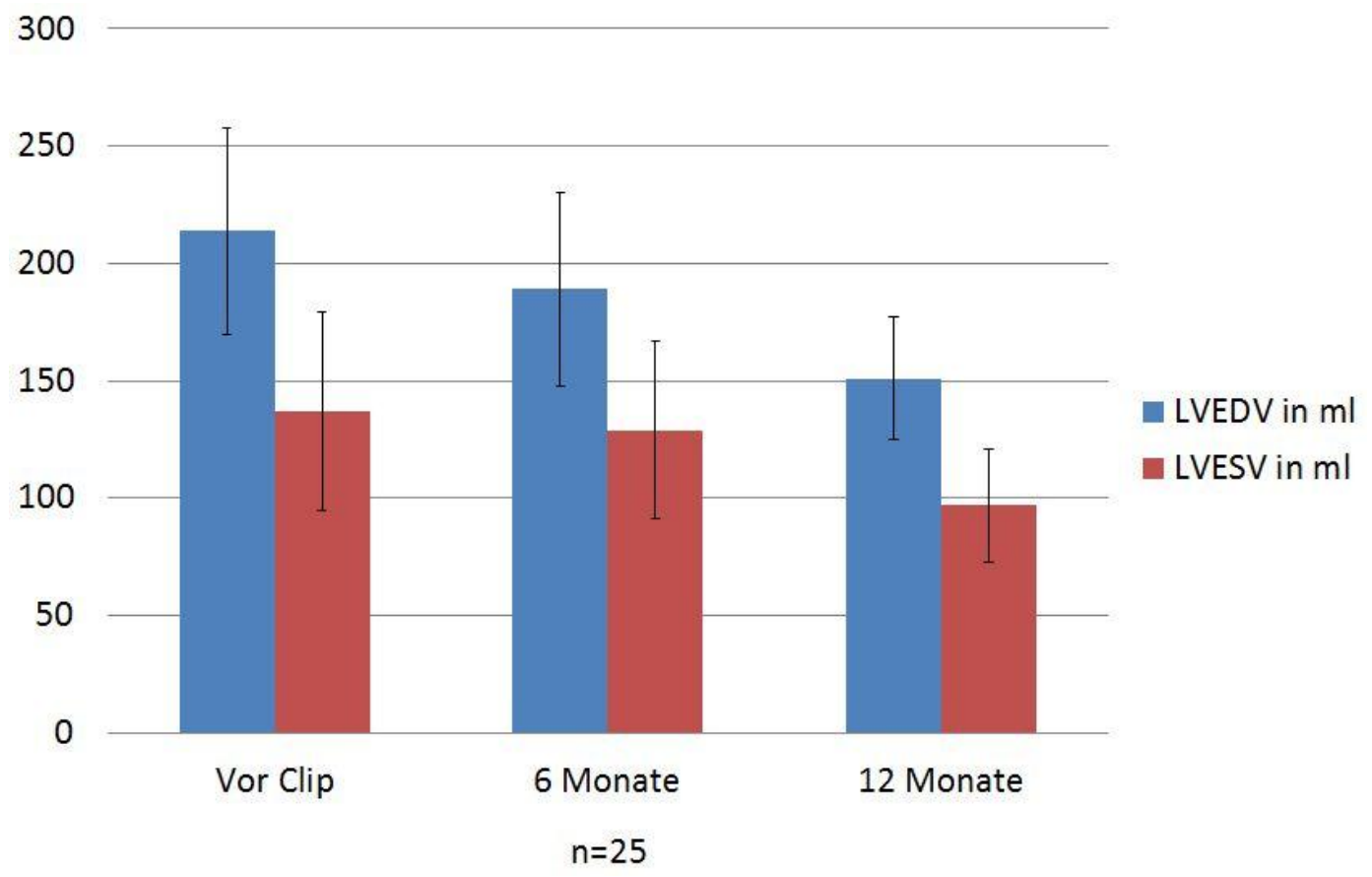

Abb. 3.6: Linksventrikuläre Volumina (enddiastolisch und endsystolisch) in $\mathrm{ml}$. Höchstsignifikante Verminderung des $\operatorname{LVEDV}(\mathrm{p}<0,001)$ und signifikante Verminderung des LVESV $(p<0,05)$ nach MitraClip ${ }^{\circledR}$-Implantation über den gesamten Beobachtungszeitraum. 


\subsection{Ejektionsfraktion über 12 Monate}

Abb. 3.7 zeigt die Ejektionsfraktion (EF) im Verlauf. Es zeigten sich vor Clip, sowie nach 6 und 12 Monaten Werte von $37 \pm 6,37 \pm 6$ und $42 \pm 7(p=0,0779)$. Es lassen sich hier keine globalen signifikanten Unterschiede $(p=0,0779)$ beobachten.

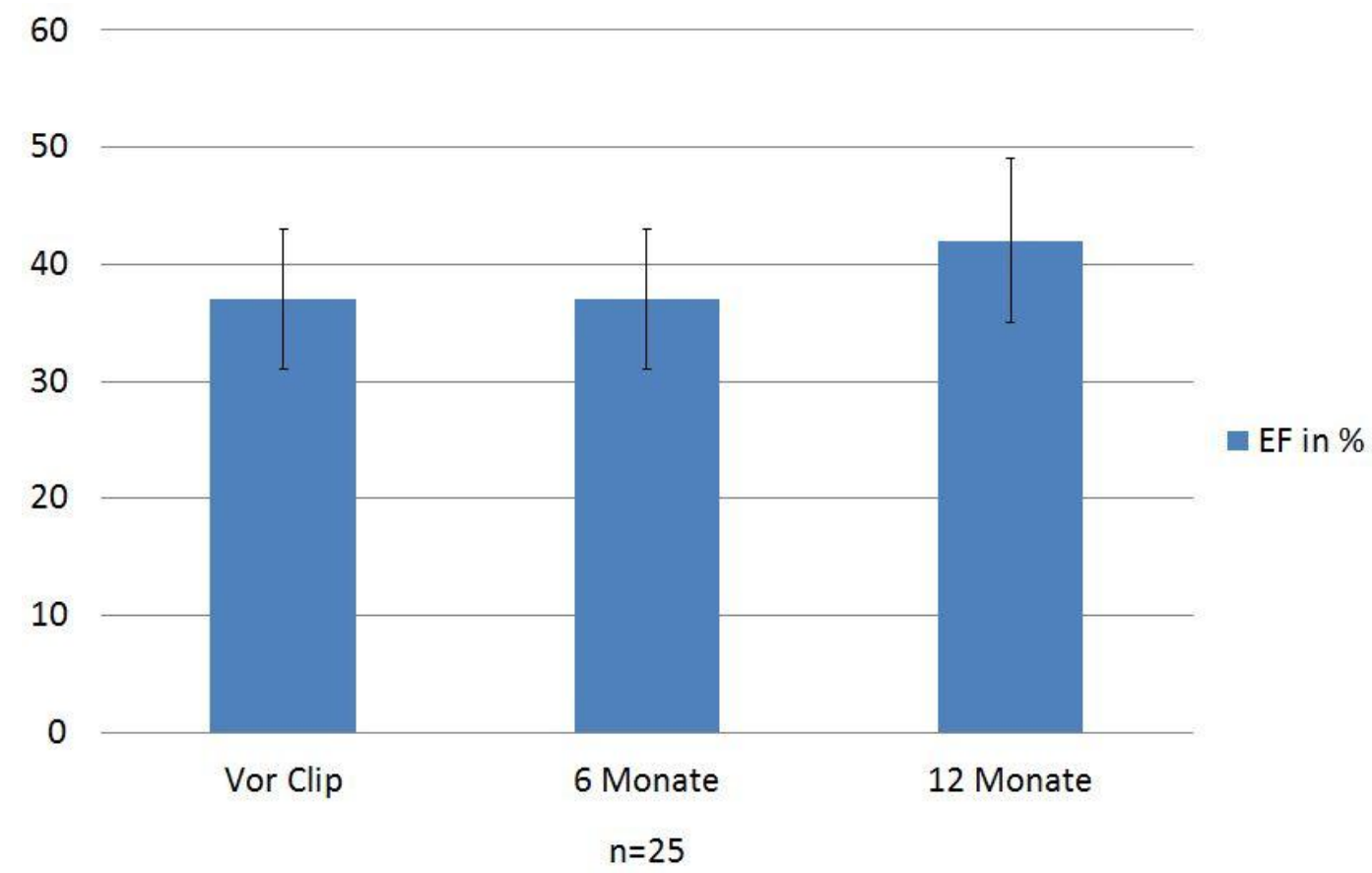

Abb. 3.7: Ejektionsfraktion (biplan nach Simpson (Lang et al. 2005)) in \%.

Keine signifikante Erhöhung der Ejektionsfraktion nach MitraClip ${ }^{\circledR}$-Implantation über den gesamten Beobachtungszeitraum. 


\subsection{NT-proBNP über 12 Monate}

Abb. 3.8 zeigt das NT-proBNP im Verlauf über 12 Monate. Es zeigten sich vor Clip, sowie nach 6 und 12 Monaten Werte von $4637 \pm 2719,3318 \pm 1544$ und $3877 \pm 1505(p=0,278)$. Es lassen sich hier keine globalen signifikanten Unterschiede $(p=0,278)$ beobachten.

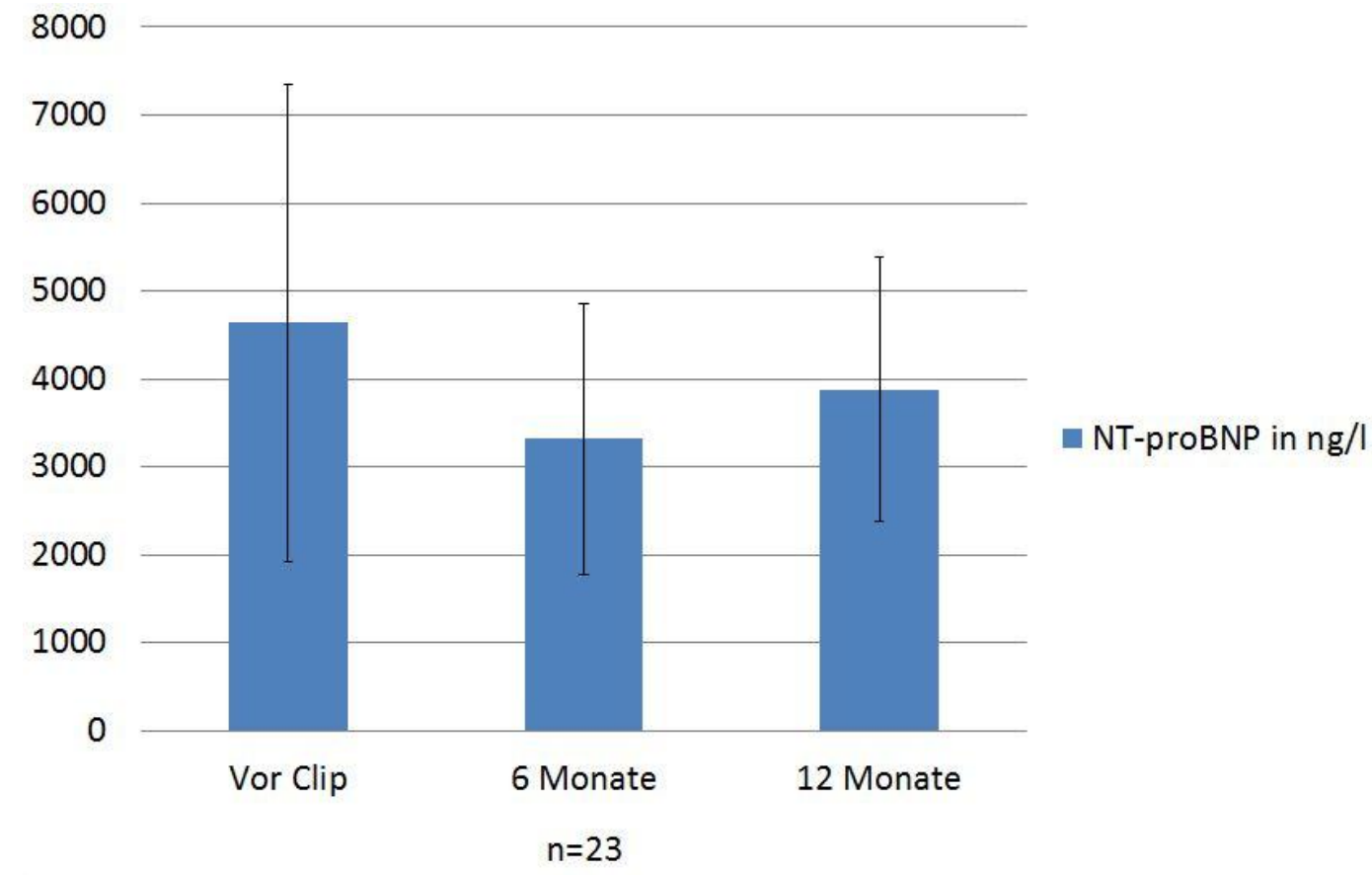

Abb. 3.8: NT-proBNP im Plasma in ng/l.

Keine signifikante Verminderung des NT-proBNP nach MitraClip ${ }^{\circledR}$-Implantation über den gesamten Beobachtungszeitraum. 


\subsection{4 Überleben}

Abb. 3.9 zeigt die Überlebenskurve der Patienten nach MitraClip ${ }^{\circledR}$-Implantation nach Kaplan-Meier. Nach 30 Tagen hatten 97,2\%, nach 6 Monaten 88,9\% und nach 12 Monaten 80,6\% der Patienten überlebt. Zum Zeitpunkt des letzten Patientenkontaktes vor Fertigstellung dieser Arbeit hatten 69,4\% aller Patienten mit einer medianen Beobachtungsdauer von 402 Tagen nach dem Eingriff überlebt. Demgegenüber steht die berechnete operative 30-Tage-Mortalität von $32 \pm 5 \%$ (logistischer EuroScore) bzw. $12 \pm 3 \%$ (STS-Score).

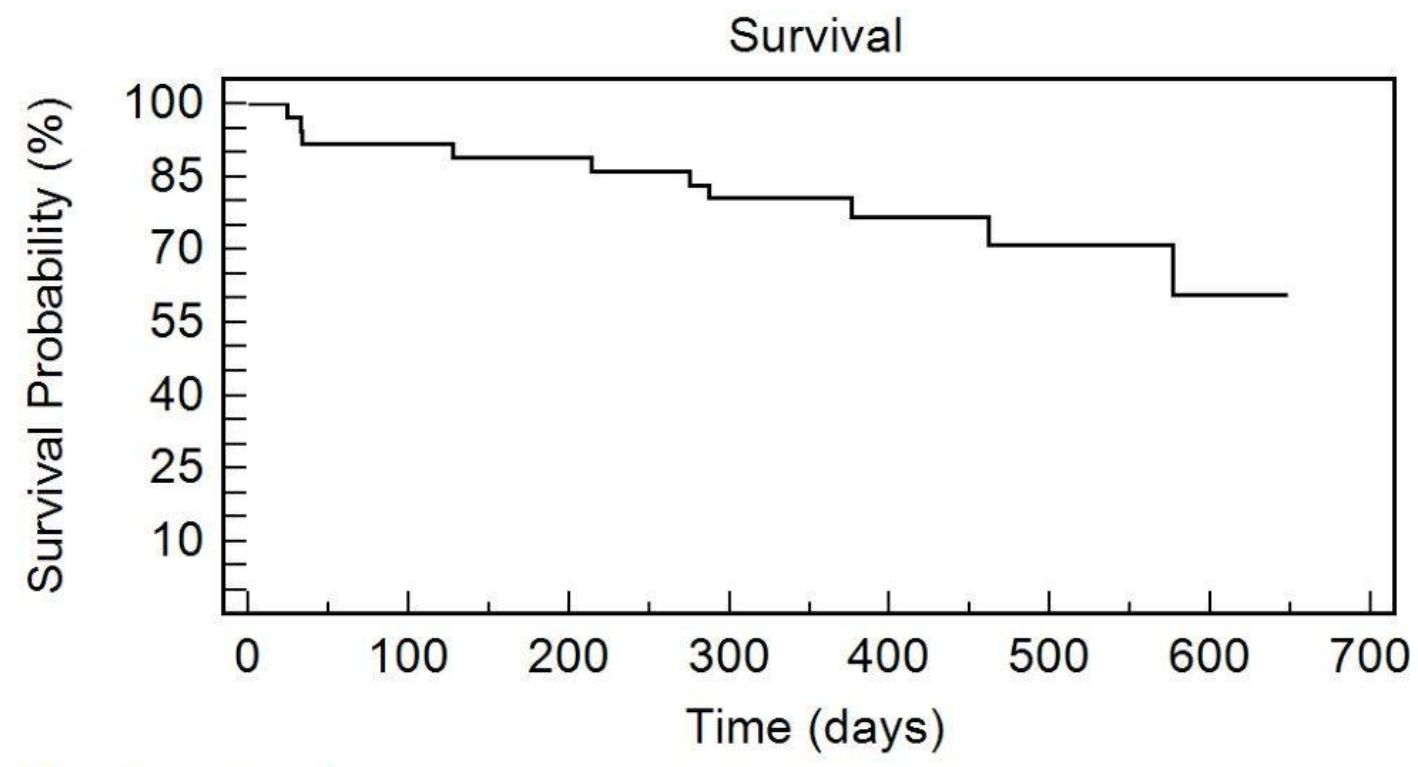

Number at risk

$\begin{array}{llllllll}36 & 33 & 32 & 29 & 18 & 10 & 5 & 1\end{array}$

Abb. 3.9: Kaplan-Meier-Überlebenskurve. Nach 30 Tagen, 6 und 12 Monaten hatten 97,2\% bzw. $88,9 \%$ bzw. 80,6\% der Patienten überlebt. 


\section{Diskussion}

\subsection{Relevanz und Aussage dieser Arbeit}

Die vorliegende Arbeit berichtet über das akute und intermediäre Outcome der bislang größten publizierten Real-World-Kohorte von Patienten mit Erreichen der 12-Monats-Follow-Up-Kontrolle, die aufgrund schwerer organischer oder funktioneller Mitralinsuffizienz aus klinischer Indikation außerhalb von kontrollierten Studien einer kathetergestützten Mitralklappen-Reparatur mit MitraClip ${ }^{\circledR}$ unterzogen wurde.

Trotz multipler Entwicklungen auf dem Gebiet kathetergestützter Methoden ist die Mitralklappenchirurgie der Goldstandard für die Behandlung der schweren symptomatischen Mitralinsuffizienz oder der asymptomatischen Mitralinsuffizienz mit reduzierter linksventrikulärer Funktion. Eine breite klinische Evidenz belegt den Nutzen der chirurgischen Therapie mit hoher Eingriffssicherheit, einer Mortalität < 5\%, langfristig sehr guter Mitralklappenfunktion und nachhaltiger Besserung von Symptomatik und Prognose (lung et al. 2003; Vahanian et al. 2007). Auf der anderen Seite ist die chirurgische Mitralklappentherapie mit einem signifikanten operativen Trauma assoziiert, birgt Komplikationsrisiken und zieht eine längere postoperative Rekonvaleszenz nach sich. Infolgedessen wird jeder dritte Patient - meist sind dies hochbetagte oder multimorbide Patienten oder Patienten mit eingeschränkter linksventrikulärer Funktion - trotz Indikation der operativen Therapie nicht unterzogen (lung et al. 2003; Mirabel et al. 2007). Aus diesem Dilemma heraus wurden in jüngerer Zeit kathetergestützte und minimal-invasive Verfahren entwickelt. Die MitraClip ${ }^{\circledR}$-Technologie (Abbott Vascular) basiert auf der Double-Orifice- oder Edge-to-Edge-RepairTechnik des italienischen Chirurgen Otavio Alfieri, der eine chirurgische Naht zur permanenten Koaptation des anterioren und posterioren Mitralsegels im mittleren Anteil im Jahre 1991 als simples und effektives Verfahren der Mitralklappen-Rekonstruktion einführte (Alfieri et al. 2001). Der MitraClip ${ }^{\circledR}$ bietet gegenüber chirurgischen Verfahren Vorteile aufgrund fehlender Notwendigkeit von Thorakotomie, Kardioplegie oder extrakorporaler Zirkulation. Der Kontraktionsablauf bleibt vollständig erhalten. Dies erlaubt eine exakte Platzierung des Clips in den Ursprung der Mitralinsuffizienz unter kontinuierlicher intraprozeduraler echokardiographischer Kontrolle und eine mehrfache Repositionierung bis zum Erzielen eines optimalen Resultates. Auch eine vollständige Entfernung des Clips bei wiederholten insuffizienten Versuchen ist möglich 
(Feldman et al. 2005; Feldman et al. 2009; Feldman et al. 2010). Hierdurch lässt sich auch das Risiko einer relevanten Mitralstenose kontrollieren (Herrmann et al. 2009). Das MitraClip ${ }^{\circledR}$-System ist allerdings nicht in der Lage eine Anuloplastie durchzuführen, die bei der operativen Rekonstruktion der Mitralklappe von den meisten Chirurgen durchgeführt wird um eventuelle Reoperationen zu vermeiden. Allerdings wurde gezeigt, dass eine Edge-to-Edge Reparatur ohne Anuloplastie den natürlichen Sphinktermechanismus der Mitralklappe in der Systole und die systolische Funktion an der Herzbasis erhält (Umana et al. 1998).

Anfang 2010 wurden 30-Tage-Outcome-Daten von 107 Patienten aus der EVEREST-Studie und der Vor-Randomisierungs-Phase der EVEREST II-Studie mit dem MitraClip ${ }^{\circledR}$ berichtet (Feldman et al. 2009). In der dortigen Kohorte waren 62\% der Patienten älter als 65 Jahre und die durchschnittliche Ejektionsfraktion betrug 62\%. Bei 74\% dieser Patienten war die Prozedur erfolgreich und 64\% wurden mit einer residualen Mitralinsuffizienz $\leq$ Grad 1 entlassen. Im März 2010 wurden die Daten der kontrollierten, randomisierten EVEREST-II-Studie auf der Tagung des American College of Cardiology in Atlanta vorgestellt (Feldman et al. 2010). Hier lag der Erfolg der Prozedur bei 77\%, nach 12 Monaten zeigten 81,5\% der Patienten eine Mitralinsuffizienz $\leq$ Grad 2. Es handelte sich bei beiden Kollektiven um Patienten mit moderatem chirurgischem Risiko und vorwiegend degenerativer Mitralinsuffizienz. Die Gruppe von Franzen aus Hamburg (Franzen et al. 2010) und gepoolte Daten zweier italienische Gruppen (Tamburino et al. 2010) zeigten kürzlich Ergebnisse von Real-World-Kohorten mit 51 bzw. 31 Risiko-Patienten und vorwiegend funktioneller Mitralinsuffizienz. Auch in diesen Kollektiven fanden sich eine hohe Eingriffssicherheit mit einer 30Tage-Mortalität von 3,2 bzw. 2,0\% und sehr gute Prozedurerfolge. Das Follow-Up war jedoch auf 30-Tage begrenzt. Andere publizierte Arbeiten fokussieren auf Patienten mit schwer reduzierter linksventrikulärer Funktion (Franzen et al. 2011) oder unzureichendem Ansprechen auf eine Resynchronisationstherapie (Auricchio et al. 2011).

Die vorliegende Arbeit berichtet erstmals über eine Real-World-Kohorte von 36 Patienten mit hohem chirurgischem Risiko und 12-monatigem Follow-Up nach MitraClip ${ }^{\circledR}$-Implantation. Die Patienten waren deutlich älter $(86,1 \%$ älter als 65 Jahre), hatten ein höheres operatives Risiko (EuroSCORE $32 \pm 6$ ) und eine weitaus geringere Ejektionsfraktion (35 $\pm 5 \%$ ), zeigten aber vergleichbare Ergebnisse mit den Patienten aus den kontrollierten EVEREST-Studien (Feldman et al. 2005; Feldman et al. 2009; Feldman et al. 2010). Daraus lässt sich schließen, dass das MitraClip ${ }^{\circledR}$ Verfahren auch bei Patienten sowohl mit hohem chirurgischen Risiko als auch mit hochgradiger LV- 
Dysfunktion durchaus sicher durchführbar ist. Die 30-Tage-Mortalität der Patienten der vorliegenden Arbeit war 2,8\%. Bei 19 Patienten (52,8\%) wurde ein MitraClip ${ }^{\circledR}$, bei 14 Patienten $(38,9 \%) 2$ MitraClips ${ }^{\circledR}$ und bei 2 Patienten (5,6\%) 3 MitraClips ${ }^{\circledR}$ erfolgreich implantiert. Bei allen erfolgreichen Eingriffen wurde die Mitralinsuffizienz um mindestens einen Grad reduziert. Bei 25 Patienten (69,4\%) wurde eine Reduktion der Mitralinsuffizienz um 2 oder 3 Grade erreicht. 83,3\% hatten nach MitraClip ${ }^{\circledR}$ eine residuale Mitralinsuffizienz $\leq$ Grad 2. Im weiteren Verlauf persistierte das Ergebnis mit 77,8\% der Patienten mit einer Mitralinsuffizienz $\leq$ Grad 2 nach 12 Monaten. Aufgrund des hohen operativen Risikos der Patienten wurde eine Mitralinsuffizienz Grad 2 (13 Patienten) und Grad 3 (5 Patienten) als akzeptables Ergebnis bewertet, da die Mitralinsuffizienz bei diesen Patienten um mindestens einen Grad reduziert wurde und der Eingriff mit einer akuten funktionellen Verbesserung einherging. Es bleibt abzuwarten, ob die Reduktion der Mitralinsuffizienz letztendlich mit einer verbesserten Langzeitprognose einhergeht. Weder nach dem Eingriff, noch im Verlauf über 12 Monate war eine Mitralklappenstenose bei einem der Patienten nachweisbar.

Die Reduktion der Mitralinsuffizienz induzierte eine signifikante mediane Verringerung der Herzinsuffizienzklasse um 1 Stadium nach NYHA. Nach 6 Monaten hatten 20 der überlebenden 32 Patienten $(62,5 \%)$ eine NYHA-Klasse von II oder weniger. Des Weiteren verbesserte sich die NYHAKlasse weiter, wie man an den Ergebnissen des FollowUp nach 12 Monaten sehen kann. Die Verbesserung der NYHA-Klasse spiegelt sich auch in den Ergebnissen des 6-Minuten-Gehtests wider. Es kam zu einer deutlich verbesserten Leistungsfähigkeit mit einer Zunahme der 6-MinutenGehstrecke um durchschnittlich 96 m innerhalb von 12 Monaten. Bei Gehstreckenveränderungen von über $70 \mathrm{~m}$ wurde nachgewiesen, dass Patienten eine deutliche Steigerung der Leistungsfähigkeit bemerkten (Redelmeier et al. 1997).

Im Minnesota Living With Heart Failure Questionnaire (MLHFQ) ist die durchschnittliche Anzahl der Punkte von 47 auf 23 (um 51\%) gefallen, was einer Halbierung der subjektiven Symptomatik entspricht. Dieses Ergebnis entspricht der Verbesserung der NYHA-Klasse und der Gehstrecke und drückt den statistisch höchstsignifikanten $(p<0,001)$ subjektiven Beschwerderückgang aus.

Wie auch in anderen Studien (Auricchio et al. 2011; Feldman et al. 2010) konnte ebenfalls in dieser Arbeit ein reverses Remodeling nachgewiesen werden. Sowohl das LVEDD, als auch die linksventrikulären Volumina LVEDV und LVESV verringerten sich signifikant. Das LVESD verringerte sich nicht signifikant. Um mehr Aussagen über das reverse Remodeling machen zu können, müsste man diese Parameter über einen längeren Zeitraum im weiteren Verlauf beobachten. 
Bezüglich einer Änderung der Ejektionsfraktion im Verlauf berichten verschiedenen Studien von unterschiedlichen Ergebnissen: Währen das italienische Team von Tamburino (Tamburino et al. 2010) keine signifikante Erhöhung der Ejektionsfraktion im Verlauf nachweisen konnte, wurde von Auricchio et. al eine statistische signifikante Verbesserung gesehen (Auricchio et al. 2011). Dies könnte an den verschiedenen Patientenkollektiven liegen. In der vorliegenden Arbeit wurde keine signifikante Besserung der Ejektionsfraktion gesehen. Um eventuelle Verbesserungen genauer untersuchen zu können, müsste man eventuell die Patienten auch hier über einen längeren Zeitraum nachuntersuchen.

Es gibt bisher keine Studie mit MitraClip, welche einen signifikanten Abfall des NT-proBNP über einen längeren Zeitraum beschrieben hat oder dieses im längeren Verlauf analysiert hat. Allerdings hat das Team von Auricchio in der Schweiz bei Patienten mit terminaler Herzinsuffizienz und erfolgloser kardialer Resynchronisationstherapie einen signifikanten Unterschied zwischen dem NTproBNP der Überlebenden im Gegensatz zu den Verstorbenen bei MitraClip-Eingriff festgestellt (Auricchio et al. 2011). In der vorliegenden Arbeit ist zwar ein Abfall des NT-proBNP über 12 Monate dokumentiert, jedoch ist dieser nicht signifikant. Fehlender Abfall von NT-proBNP im Rahmen einer Herzinsuffizienzbehandlung wurde in früheren Arbeiten als Prädiktor für eine ungünstige Langzeitprognose gefunden (O'Donoghue und Braunwald 2010). Die Bedeutung dieses Befundes für das untersuchte Hochrisikokollektiv der vorliegenden Arbeit müssen Langzeitbeobachtungen klären.

Die 30-Tage-Mortalität von $2,8 \%$ in dieser Arbeit steht einer berechneten Mortalität von $32 \pm 5 \%$ (logistischer EuroScore) bzw. $12 \pm 3 \%$ (STS-Score) für eine konventionelle Herzklappenoperation gegenüber. Die Validität von Risiko-Scores zur Abschätzung des operativen Risikos von Herzklappeneingriffen bei Hochrisikopatienten wird zwar kontrovers diskutiert. Einige Autoren berichteten, dass das tatsächliche Risiko der Operation durch Scores bis zu 3-fach überschätzt wurde (Mack 2011). Trotzdem lassen die vorliegenden Befunde den Schluss zu, dass der MitraClip ${ }^{\circledR}$ Eingriff auch im Vergleich zur konventionellen Mitralklappenchirurgie als sehr sicher betrachtet werden kann. Zum selben Schluss kam auch die kontrollierte, randomisierte EVEREST-II-Studie (Feldman et al. 2010). Sowohl die Mortalität nach 6 Monaten (11,1 \%) und nach 12 Monaten (19,4 \%) zeigt, dass diese Sicherheit auch über einen längeren Zeitraum gegeben ist. Ähnliche Mortalitätsraten wie die hier gezeigten, besonders für die 30-Tage-Mortalität, wurden ebenfalls in anderen Studien gezeigt (Auricchio et al. 2011; Feldman et al. 2010; Franzen et al. 2010; Tamburino et al. 2010). Zum Zeitpunkt des letzten Patientenkontaktes vor Fertigstellung dieser Arbeit hatten 
69,4\% aller Patienten mit einer medianen Beobachtungsdauer von 402 Tagen nach dem Eingriff überlebt.

Zusammenfassend zeigt MitraClip ${ }^{\circledR}$ sehr gute intermediäre Ergebnisse in einer Real-World-Kohorte von Patienten mit hohem chirurgischem Risiko. Gerade diese Patientengruppe, welche sonst aufgrund von Risikofaktoren nur sehr limitierte Therapieoptionen hat, profitiert klinisch von der Reduktion der Mitralinsuffizienz. MitraClip ${ }^{\circledR}$ stellt für diese Patienten eine sichere und effektive Alternative zur Operation dar.

\subsection{Ausblick}

Langzeituntersuchungen und große kontrollierte, randomisierte Studien müssen folgen, um den Stellenwert des MitraClip-Verfahrens im Vergleich zur klassischen Mitralklappenchirurgie und zu neuen, minimal-invasiven chirurgischen Verfahren zu determinieren. Bis diese Daten vorliegen, gilt die klassische Mitralklappenchirurgie unverändert als Goldstandard für Patienten mit schwerer Mitralinsuffizienz. Das MitraClip ${ }^{\circledR}$-Verfahren stellt aber eine neue, zusätzliche Therapieoption für Patienten dar, welche bisher aufgrund von Komorbiditäten für eine chirurgische Intervention nicht in Frage kamen.

Ferner könnten durch die technische Weiterentwicklung des MitraClip ${ }^{\circledR}$-Systems, wie zum Beispiel eine Verlängerung der Greifarme, sowohl die Rate der erfolgreichen Implantationen als auch die Ergebnisse des Eingriffs verbessert werden. Hiervon wurde sowohl in unserem, als auch in anderen Zentren berichtet (Franzen et al. 2010).

Durch die weitere Evaluation dieses Verfahrens wird es in Zukunft möglich sein, das Patientenkollektiv besser zu identifizieren, welches von dem Eingriff profitiert.

Als Ergänzung kann die transösophageale 3-D-Echokardiographie aufgrund der hervorragenden Visualisierung nicht nur die transseptale Punktion und das Positionieren des Clips über dem maximalen Insuffizienzjet, sondern auch das Greifen der Mitralsegel und die Evaluation des Endergebnisses effizienter und sicherer machen. Hierdurch könnte eine deutliche Reduktion der Eingriffszeiten und folglich eine weitere Erhöhung der Eingriffssicherheit erreicht werden (Altiok et al. 2011; Swaans et al. 2009). Auch ist es vorstellbar, dass gerade durch die transösophageale 3-DEchokardiographie eine noch bessere Evaluation vor MitraClip ${ }^{\circledR}$-Eingriff möglich sein wird. 


\subsection{Fazit}

- Die kathetergestützte Mitralklappen-Rekonstruktion mit MitraClip ${ }^{\circledR}$ zeigt eine hohe Eingriffssicherheit und sehr gute Ergebnisse direkt nach Intervention sowie in der Nachkontrolle über mindestens 12 Monate.

- Das MitraClip ${ }^{\circledR}$-System stellt eine Therapieoption für Patienten mit schwerer organischer und funktioneller Mitralinsuffizienz und hohem operativem Risiko dar.

- Die Therapie sollte an einem Zentrum mit Erfahrung in der Therapie schwer herzinsuffizienter Patienten durchgeführt werden und stellt eine Zusatzoption bei Ausschöpfen der Standardtherapie dar.

- Die Indikation für diese Therapie sollte durch ein Heart Team bestehend aus je einem erfahrenen interventionellen und konservativen Kardiologen und Herzchirurgen befürwortet werden.

- Der langfristige Stellenwert des Verfahrens muss durch weiterführende und größere randomisierte Studien determiniert werden. 


\section{Zusammenfassung}

Hintergrund: Publizierte Verlaufskontrollen nach MitraClip ${ }^{\circledR}$-Implantation in Real-World-Kohorten sind auf das 30-Tage-Outcome beschränkt.

Material und Methoden: Die ersten 36 konsekutiven Patienten unseres Zentrums mit 12monatigem Verlauf nach MitraClip ${ }^{\circledR}$-Implantation wurden klinischen und echokardiographischen Kontrollen unterzogen.

Ergebnisse: Es handelte sich um Patienten im Alter von $73 \pm 3$ Jahren mit signifikanter Mitralinsuffizienz und hohem Mortalitätsrisiko für die klassische Mitralklappenchirurgie (log. EuroScore $32 \pm 6 \%$, STS-Score $12 \pm 3 \%$ ). Der MitraClip ${ }^{\circledR}$-Eingriff erwies sich als sehr sicher mit einer 30-Tage-Mortalität von 2,8\%. Es traten keine Fälle von Myokardinfarkt, Schlaganfall oder Perikardtamponade auf. In keinem Fall war eine Notfall-Operation oder mechanische Kreislaufunterstützung notwendig. 8,3\% erlitten hämodynamisch relevante Blutungen. Bei 8,3\% kam es zu einer partiellen Cliplösung von einem Segel. Der Eingriff war effektiv mit einem Prozedurerfolg von 97,2\% und einer medianen Reduktion der Mitralinsuffizienz um 2 Schweregrade. 83,3\% der Patienten zeigten nach dem Eingriff eine residuale Mitralinsuffizienz von $\leq 2$. Das Ergebnis war in den 6- und 12-Monats-Kontrollen stabil. Die 6-Minuten-Gehstrecke nahm innerhalb von 12 Monaten um durchschnittlich $96 \mathrm{~m}$ zu. Das 6- und 12-Monatsüberleben war 88,9 bzw. $80,6 \%$.

Schlussfolgerung: MitraClip ${ }^{\circledR}$ zeigte sehr gute akute und intermediäre Ergebnisse in einer RealWorld-Kohorte und stellt eine sichere Therapieoption für Patienten mit schwerer Mitralinsuffizienz und erhöhtem OP-Risiko dar. 


\section{Anhänge}

\subsection{Anhang 1: Evaluationsbogen}

\section{Evalution für Mitralklappenrekonstruktion mit MitraClip ${ }^{\top M}$}

\begin{tabular}{|c|c|}
\hline $\begin{array}{ll} & 07.01 .1934 \\
& 75 \text { Jahre } \\
: & 174 \mathrm{~cm}, 93 \mathrm{~kg} \\
\text { : } & \text { BMI } 32 \mathrm{~kg} / \mathrm{m}^{2}, \mathrm{E} \\
\text { : } & \text { Mitralinsuffizien } \\
\quad & \text { Symptome } \\
& -\quad \text { NYHA I }\end{array}$ & $\begin{array}{l}\text { 4, *, \#02994054 Zuweiser: Info postinterv. D } \\
\text { BSA 2,1 } \text { m² }_{\text {IZ }}^{\square} \text { funktionell a degenerativ } \\
\text { IV }\end{array}$ \\
\hline 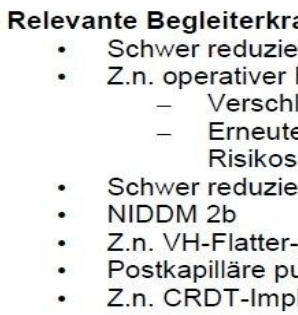 & $\begin{array}{l}\text { ankungen } \\
\text { erter LV-Funktion, EF } 30 \% \\
\text { Revaskularisation ' } 82 \text { sowie ' } 90 \\
\text { luss aller venöser Grafts } \\
\text { e operative Revask. in } 07 / 05 \text { auf der Herzkonferenz diskutiert, aber wg. hohen } \\
\text {, ausgedehnter Narbenareale und Ablehnung des Pat. abgelehnt } \\
\text { erte LV-Funktion } \\
\text {-Ablation } \\
\text { ulmonale Hypertonie } \\
\text { lantation '05 }\end{array}$ \\
\hline $\begin{array}{l}\text { Echokardiographie TT } \\
\text { LV-Funktion, EF } \\
\text { - } \text { LVED/ES } 58 /-n \\
\text { IVS/PW } 8 / 8 \mathrm{~mm} \\
\text { Morphologie: fu } \\
\text { leicht nach med } \\
\text { post. Wand ent } \\
\text { : Koadaptationsti } \\
\text { : } \text { Koadaptionslän } \\
\text { "Flail Gap", Pro } \\
\text { MI III }, \Delta \mathrm{P}_{\text {mittl }} 1 \\
\text { EROA } \mathrm{cm}^{2}, V_{\mathrm{R}} \\
\text { : PV-Fluss abgef } \\
\text { AK: } \mathrm{m} / \mathrm{s}, \mathrm{Al}^{\circ}, \mathrm{T} \\
\text { Sonstiges: }\end{array}$ & $\begin{array}{l}\text { TE/TEE } 24.04 .09 \\
\text { F } 30 \% \\
\text { mm, LA } 51 \mathrm{~mm} \\
\text { unktionell. Dilatierter Anulus, wenig Koadaptation, leichter Prolaps des AMS, } \\
\text { diale reichende, gering dezentrale MI zwischen A2/(A3) und P2(P3) an der } \\
\text { lang bei AMS-Prolaps } \\
\text { iefe (4C, größte Tiefe) } \mathrm{mm} \\
\text { ge (4C, kürzeste Länge) } \mathrm{mm} \\
\text { lapsspaltgröße (LAX, } 4 \mathrm{C}, 5 \mathrm{C} \text {, größter Spalt) } \mathrm{mm} \\
\mathrm{mm} \mathrm{Hg}, \mathrm{MOOF} \mathrm{cm}^{2} \\
\text { leg } 108 \mathrm{ml}, \mathrm{F}_{\text {Reg }} 64 \%, \mathrm{VC} 0,588 \mathrm{~cm} \\
\text { flacht }\end{array}$ \\
\hline $\begin{array}{c}\text { Linksherzkatheter } 07 / C \\
\cdot \quad \text { Verschluß aller }\end{array}$ & 05 Grafts, MRT (07/05) Narbe Cx-Areal, sowie midinferior bis inferoapikal (ACD) \\
\hline $\begin{array}{l}\text { Hämodynamik NA } \\
: \text { LVP } \\
: \text { AoP } \\
: \text { PAP } \\
: \text { PCWP } \\
\text { Cl }\end{array}$ & $\begin{array}{l}\mathrm{mm} \mathrm{Hg} \\
\mathrm{mm} \mathrm{Hg} \\
\mathrm{mm} \mathrm{Hg} \\
\mathrm{mm} \mathrm{Hg} \\
\mathrm{L} / \mathrm{min} / \mathrm{m}^{2}\end{array}$ \\
\hline $\begin{array}{l}\text { Sonstige relevante Be } \\
\cdot \quad \mathrm{Krea} 3,1 \mathrm{mg} / \mathrm{dl}\end{array}$ & funde \\
\hline $\begin{array}{l}\text { Berechnete 30-d-Mort } \\
\text { - Logistischer Eu }\end{array}$ & $\begin{array}{l}\text { alität für chirurgischen Mitralklappenersatz bzw. -rekonstruktion } \\
\text { roScore } 60 \% \text {, STS Score } 18 \%\end{array}$ \\
\hline $\begin{array}{c}\text { Bemerkung/Sonstiges } \\
\text { • Kein Linksherzk }\end{array}$ & katheter wg. schwerer Niereninsuffizienz \\
\hline $\begin{array}{l}\text { Indikation } \\
\square \text { MitraClip }^{\mathrm{TM}}\end{array}$ & 口MKR \\
\hline
\end{tabular}




\subsection{Anhang 2: EuroSCORE}

5

EurosCORE Risk Profile

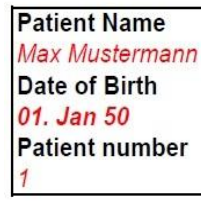

Operation

Mitral Valve Replacement

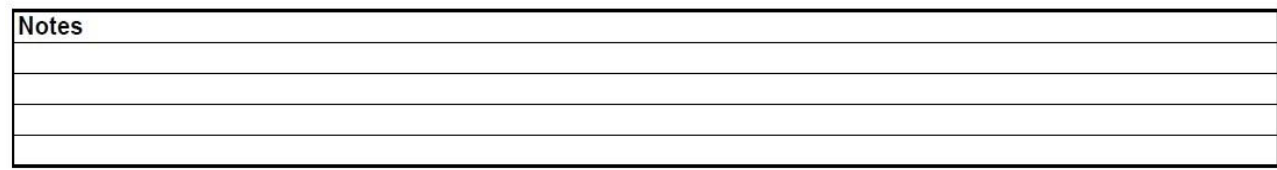

\begin{tabular}{|c|c|c|c|}
\hline & & $\begin{array}{c}\text { Additive } \\
\text { EurosCORE } \\
\Phi\end{array}$ & $\begin{array}{c}\text { Logistic } \\
\text { EuroSCORE } \\
\beta \mathrm{i} \mathrm{Xi}\end{array}$ \\
\hline \multicolumn{4}{|l|}{ Patient Factors } \\
\hline $\begin{array}{l}\text { Age } \\
\text { Sex } \\
\text { Chronic pulmonary disease } \\
\text { Extracardiac arteriopathy } \\
\text { Neurological dysfunction } \\
\text { Previous cardiac surgery } \\
\text { Serum creatinine }>200 \mu \mathrm{mol} / \mathrm{L} \\
\text { Active endocarditis } \\
\text { Critical preoperative state }\end{array}$ & $\begin{array}{l}61 \mathrm{yr} \\
\begin{array}{l}\square \text { Female } \\
\square \text { Yes } \\
\square \text { Yes } \\
\square \text { Yes } \\
\square \text { Yes } \\
\square \text { Yes } \\
\square \text { Yes } \\
\square \text { Yes }\end{array}\end{array}$ & $\begin{array}{l}1 \\
1 \\
\\
3 \\
2\end{array}$ & $\begin{array}{c}0,214693782 \\
0,4931341 \\
1,002625 \\
0,6521653\end{array}$ \\
\hline \multicolumn{4}{|l|}{ Cardiac Factors } \\
\hline $\begin{array}{l}\text { Unstable angina } \\
\text { LV dysfunction moderate or LVEF } 30-50 \% \\
\text { LV dysfunction poor or LVEF }<30 \\
\text { Recent myocardial infarct } \\
\text { Pulmonary hypertension }\end{array}$ & $\begin{array}{ll}\square & \text { Yes } \\
\square & \text { Moderate } \\
\square & \text { Poor } \\
\square & \text { Yes } \\
\square & \text { Yes }\end{array}$ & $\begin{array}{l}3 \\
2\end{array}$ & $\begin{array}{l}1,094443 \\
0,7676924\end{array}$ \\
\hline \multicolumn{4}{|l|}{ Operation Factors } \\
\hline $\begin{array}{l}\text { Emergency } \\
\text { Other than isolated CABG } \\
\text { Surgery on thoracic aorta } \\
\text { Postinfarct septal rupture }\end{array}$ & $\begin{array}{l}\square \text { Yes } \\
\square \quad \text { Yes } \\
\square \text { Yes } \\
\square \text { Yes }\end{array}$ & & \\
\hline$B^{*}$ EuroscoRE & & $\Sigma \Phi$ & $\mathrm{e}^{\left(-4.789594+\sum \beta_{i} X_{i}\right)} / 1+e^{\left(-4.789594+\sum \beta_{i} X_{i}\right)}$ \\
\hline Downloaded from http://euroscore.org & & 12 & $36,24 \%$ \\
\hline
\end{tabular}




\subsection{Anhang 3: Minnesota Living With Heart Failure Questionnaire (MLHFQ)} Bitte kreisen Sie bei den folgenden Fragen ein, inwieweit Ihre Herzkrankheit Ihr Leben in den letzten 4
Wochen beeinträchtigt hat. Wenn eine Frage nicht auf Sie zutrifft, kreisen Sie bitte die 0 ein.

Nein Selten Immer

1. Traten Schwellungen an den Fußknöcheln, Beinen etc. auf?

$\begin{array}{llllll}0 & 1 & 2 & 3 & 4 & 5\end{array}$

2. Mussten Sie sich tagsüiber hinsetzen oder hinlegen um sich auszuruhen?

\begin{tabular}{llllll}
0 & 1 & 2 & 3 & 4 & 5 \\
\hline
\end{tabular}

3. Hatten Sie Schwierigkeiten beim Umhergehen oder Treppensteigen?

\begin{tabular}{llllll}
0 & 1 & 2 & 3 & 4 & 5 \\
\hline
\end{tabular}

4. Hatten Sie Schwierigkeiten bei Erledigungen im Haus oder im Garten?

\begin{tabular}{llllll}
0 & 1 & 2 & 3 & 4 & 5 \\
\hline
\end{tabular}

5. Hatten Sie Schwierigkeiten sich von zuhause wegzubewegen?

$\begin{array}{llllll}0 & 1 & 2 & 3 & 4 & 5\end{array}$

6. Hatten Sie Schwierigkeiten nachts gut zu schlafen?

$\begin{array}{llllll}0 & 1 & 2 & 3 & 4 & 5\end{array}$

7. Hatten Sie Schwierigkeiten im Zusammenleben oder gemeinsamen Tätigkeiten mit Freunden \begin{tabular}{llllllll} 
oder der Familie? & 0 & 1 & 2 & 3 & 4 & 5 \\
\hline
\end{tabular}

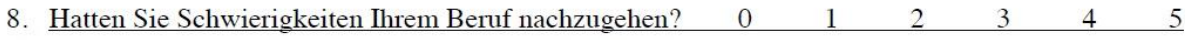

9. Hatten Sie Schwierigkeiten bei Ihrer Freizeitgestaltung, beim Sport oder ihren Hobbys? $\quad$\begin{tabular}{lllllll}
0 & 1 & 2 & 3 & 4 & 5 \\
\hline
\end{tabular}

10. Waren Sie bei Ihren sexuellen Aktivitäten beeinträchtigt?

\begin{tabular}{llllll}
0 & 1 & 2 & 3 & 4 & 5 \\
\hline
\end{tabular}

11. Konnten Sie von den Nahrungsmitteln, die Sie gerne mögen, nur weniger essen? \begin{tabular}{llllll}
0 & 1 & 2 & 3 & 4 & 5 \\
\hline
\end{tabular}

12. Waren Sie kurzatmig? \begin{tabular}{llllll}
0 & 1 & 2 & 3 & 4 & 5 \\
\hline
\end{tabular}

13. Waren Sie müde, abgeschlagen oder antriebsarm? \begin{tabular}{llllll}
0 & 1 & 2 & 3 & 4 & 5 \\
\hline
\end{tabular}

14. Mussten Sie in einem Krankenhaus stationär aufgenommen werden?

\begin{tabular}{llllll}
0 & 1 & 2 & 3 & 4 & 5 \\
\hline
\end{tabular}

15. Mussten Sie wegen Ihrer Herzerkrankung Geld ausgeben? \begin{tabular}{llllll}
0 & 1 & 2 & 3 & 4 & 5 \\
\hline
\end{tabular}

16. Hatten Sie Medikamentennebenwirkungen? \begin{tabular}{llllll}
0 & 1 & 2 & 3 & 4 & 5 \\
\hline
\end{tabular}

17. Hatten Sie den Eindruck, dass Sie für Ihre Familie oder Freunde eine Belastung sind?

\begin{tabular}{llllll}
0 & 1 & 2 & 3 & 4 & 5 \\
\hline
\end{tabular}

18. Hatten Sie den Eindruck, dass Sie Ihr Leben nicht mehr selbst bestimmen können?

\begin{tabular}{llllll}
0 & 1 & 2 & 3 & 4 & 5 \\
\hline
\end{tabular}

19. Haben Sie sich Sorgen gemacht?

\begin{tabular}{llllll}
0 & 1 & 2 & 3 & 4 & 5 \\
\hline
\end{tabular}

20. Hatten Sie Schwierigkeiten sich zu konzentrieren oder sich an Dinge zu erinnern? \begin{tabular}{llllll}
0 & 1 & 2 & 3 & 4 & 5 \\
\hline
\end{tabular}

21. Hatten Sie Zeiten, in denen Sie ohne äußeren Anlass traurig und nicht aufzuheitern waren?

$\begin{array}{llllll}0 & 1 & 2 & 3 & 4 & 5\end{array}$




\section{Literaturverzeichnis}

Abbott-Vascular (2008): MitraClip Mitral Valve Repair System. Abbott (www.abbottvascular.com)

Abbott-Vascular (2010): MitraClip Positioning and Imaging Guide. Abbott (www.abbottvascular.com)

Akins CW, Hilgenberg AD, Buckley MJ, Vlahakes GJ, Torchiana DF, Daggett WM, Austen WG (1994): Mitral valve reconstruction versus replacement for degenerative or ischemic mitral regurgitation. Ann Thorac Surg 58, 668-675; discussion 675-666

Alfieri O, Maisano F (1999): An effective technique to correct anterior mitral leaflet prolapse. J Card Surg $\underline{14}, 468-470$

Alfieri O, Maisano F, De Bonis M, Stefano PL, Torracca L, Oppizzi M, La Canna G (2001): The doubleorifice technique in mitral valve repair: a simple solution for complex problems. J Thorac Cardiovasc Surg $\underline{122}, 674-681$

Altiok E, Becker M, Hamada S, Reith S, Marx N, Hoffmann R (2011): Optimized guidance of percutaneous edge-to edge repair of the mitral valve using real-time 3-D transesophageal echocardiography. Clin Res Cardiol, (im Druck)

Alvarez JM, Deal CW, Loveridge K, Brennan P, Eisenberg R, Ward M, Bhattacharya K, Atkinson SJ, Choong C (1996): Repairing the degenerative mitral valve: ten- to fifteen-year follow-up. J Thorac Cardiovasc Surg $\underline{112}, 238-247$

Auricchio A, Schillinger W, Meyer S, Maisano F, Hoffmann R, Ussia G, Pedrazzini G, van der Heyden J, Fratini S, Klersy C, et al. (2011): Correction of mitral regurgitation in non-responders to cardiac resynchronization therapy by MitraClip improves symptoms and promotes reverse remodeling. Eur Heart J, (im Druck)

Bhalla V, Willis S, Maisel AS (2004): B-type natriuretic peptide: the level and the drug--partners in the diagnosis of congestive heart failure. Congest Heart Fail $\underline{10}$, 3-27

Bonow RO, Carabello B, de Leon AC, Jr., Edmunds LH, Jr., Fedderly BJ, Freed MD, Gaasch WH, McKay CR, Nishimura RA, O'Gara PT, et al. (1998): Guidelines for the management of patients with valvular heart disease: executive summary. A report of the American College of Cardiology/American Heart Association Task Force on Practice Guidelines (Committee on Management of Patients with Valvular Heart Disease). Circulation 98, 1949-1984

Burkart C, Turina J, Lüscher T, Hellerman J (2006): Lebensqualität von ambulanten kardiologischen Patienten einer Universitätsklinik. Kardiovask Med $\underline{9}$, 68-76

Carpentier A (1983): Cardiac valve surgery--the "French correction". J Thorac Cardiovasc Surg 86, 323337 
Cohn LH, Couper GS, Aranki SF, Rizzo RJ, Kinchla NM, Collins JJ, Jr. (1994): Long-term results of mitral valve reconstruction for regurgitation of the myxomatous mitral valve. J Thorac Cardiovasc Surg 107, 143-150; discussion 150-141

Enriquez-Sarano M, Schaff HV, Orszulak TA, Tajik AJ, Bailey KR, Frye RL (1995): Valve repair improves the outcome of surgery for mitral regurgitation. A multivariate analysis. Circulation $\underline{91}, 1022-1028$

Enriquez-Sarano M, Schaff HV, Frye RL (2003): Mitral regurgitation: what causes the leakage is fundamental to the outcome of valve repair. Circulation 108, 253-256

Erbel R, Kahlert P, Plicht B, Konorza T (2009): Mitralklappeninsuffizienz. Herz 34, 423-425

Feldman T, Wasserman HS, Herrmann HC, Gray W, Block PC, Whitlow P, St Goar F, Rodriguez L, Silvestry F, Schwartz A, et al. (2005): Percutaneous mitral valve repair using the edge-to-edge technique: sixmonth results of the EVEREST Phase I Clinical Trial. J Am Coll Cardiol 46, 2134-2140

Feldman T, Kar S, Rinaldi M, Fail P, Hermiller J, Smalling R, Whitlow PL, Gray W, Low R, Herrmann HC, et al. (2009): Percutaneous mitral repair with the MitraClip system: safety and midterm durability in the initial EVEREST (Endovascular Valve Edge-to-Edge REpair Study) cohort. J Am Coll Cardiol 54, 686-694

Feldman T, Mauri L, Foster E, Glower D: Endovascular Valve Edge-to-Edge Repair Study (EVEREST II) Randomized Clinical Trial: Primary Safety and Efficacy Endpoints. American College of Cardiology Meeting, Atlanta/GA, 2010

Franzen O, Baldus S, Rudolph V, Meyer S, Knap M, Koschyk D, Treede H, Barmeyer A, Schofer J, CostardJackle A, et al. (2010): Acute outcomes of MitraClip therapy for mitral regurgitation in high-surgical-risk patients: emphasis on adverse valve morphology and severe left ventricular dysfunction. Eur Heart J $\underline{31}$, 1373-1381

Franzen O, van der Heyden J, Schlüter M, Baldus S, Schillinger W, Butter C, Hoffmann R, Corti R, Pedrazzini G, Swaans M, et al. (2011): MitraClip therapy in patients with end-stage systolic heart failure. Eur Heart J (im Druck)

Fucci C, Sandrelli L, Pardini A, Torracca L, Ferrari M, Alfieri O (1995): Improved results with mitral valve repair using new surgical techniques. Eur J Cardiothorac Surg 9 , 621-626 discuss 626-627

Galloway AC, Colvin SB, Baumann FG, Grossi EA, Ribakove GH, Harty S, Spencer FC (1989): A comparison of mitral valve reconstruction with mitral valve replacement: intermediate-term results. Ann Thorac Surg $\underline{47}, 655-662$

Gillinov AM, Cosgrove DM, Blackstone EH, Diaz R, Arnold JH, Lytle BW, Smedira NG, Sabik JF, McCarthy PM, Loop FD (1998): Durability of mitral valve repair for degenerative disease. J Thorac Cardiovasc Surg $\underline{116}, 734-743$

Gillinov AM, Wierup PN, Blackstone EH, Bishay ES, Cosgrove DM, White J, Lytle BW, McCarthy PM (2001): Is repair preferable to replacement for ischemic mitral regurgitation? J Thorac Cardiovasc Surg $\underline{122}, 1125-1141$ 
Graves EJ, Owings MF (1997): 1995 summary: National Hospital Discharge Survey. Adv Data, 1-10

Grigioni F, Enriquez-Sarano M, Ling LH, Bailey KR, Seward JB, Tajik AJ, Frye RL (1999): Sudden death in mitral regurgitation due to flail leaflet. J Am Coll Cardiol 34, 2078-2085

Grossi EA, Goldberg JD, LaPietra A, Ye X, Zakow P, Sussman M, Delianides J, Culliford AT, Esposito RA, Ribakove GH, et al. (2001): Ischemic mitral valve reconstruction and replacement: comparison of longterm survival and complications. J Thorac Cardiovasc Surg 122, 1107-1124

GUSTO-Investigators (1993): An international randomized trial comparing four thrombolytic strategies for acute myocardial infarction. The GUSTO investigators. N Engl J Med 329, 673-682

Herrmann HC, Kar S, Siegel R, Fail P, Loghin C, Lim S, Hahn R, Rogers JH, Bommer WJ, Wang A, et al. (2009): Effect of percutaneous mitral repair with the MitraClip device on mitral valve area and gradient. Eurolntervention $\underline{4}, 437-442$

lung B, Baron G, Butchart EG, Delahaye F, Gohlke-Barwolf C, Levang OW, Tornos P, Vanoverschelde JL, Vermeer F, Boersma E, et al. (2003): A prospective survey of patients with valvular heart disease in Europe: The Euro Heart Survey on Valvular Heart Disease. Eur Heart J 24, 1231-1243

Kron IL, Green GR, Cope JT (2002): Surgical relocation of the posterior papillary muscle in chronic ischemic mitral regurgitation. Ann Thorac Surg $\underline{74}, 600-601$

Lang RM, Bierig M, Devereux RB, Flachskampf FA, Foster E, Pellikka PA, Picard MH, Roman MJ, Seward J, Shanewise JS, et al. (2005): Recommendations for chamber quantification: a report from the American Society of Echocardiography's Guidelines and Standards Committee and the Chamber Quantification Writing Group, developed in conjunction with the European Association of Echocardiography, a branch of the European Society of Cardiology. J Am Soc Echocardiogr 18, 1440-1463

Lawrie GM (1998): Mitral valve repair vs replacement. Current recommendations and long-term results. Cardiol Clin $\underline{16}, 437-448$

Levey AS, Bosch JP, Lewis JB, Greene T, Rogers N, Roth D (1999): A more accurate method to estimate glomerular filtration rate from serum creatinine: a new prediction equation. Modification of Diet in Renal Disease Study Group. Ann Intern Med 130, 461-470

Lorusso R, Borghetti V, Totaro P, Parrinello G, Coletti G, Minzioni G (2001): The double-orifice technique for mitral valve reconstruction: predictors of postoperative outcome. Eur J Cardiothorac Surg 20, 583589

Mack MJ (2011): Risk scores for predicting outcomes in valvular heart disease: how useful? Curr Cardiol Rep 13, 107-112

Maisano F, Torracca L, Oppizzi M, Stefano PL, D'Addario G, La Canna G, Zogno M, Alfieri O (1998): The edge-to-edge technique: a simplified method to correct mitral insufficiency. Eur J Cardiothorac Surg $\underline{13}$, 240-245; discussion 245-246 
Maisano F, Schreuder JJ, Oppizzi M, Fiorani B, Fino C, Alfieri O (2000): The double-orifice technique as a standardized approach to treat mitral regurgitation due to severe myxomatous disease: surgical technique. Eur J Cardiothorac Surg 17, 201-205

Mirabel M, lung B, Baron G, Messika-Zeitoun D, Detaint D, Vanoverschelde JL, Butchart EG, Ravaud P, Vahanian A (2007): What are the characteristics of patients with severe, symptomatic, mitral regurgitation who are denied surgery? Eur Heart J $\underline{28}$, 1358-1365

Nashef SA, Roques F, Michel P, Gauducheau E, Lemeshow S, Salamon R (1999): European system for cardiac operative risk evaluation (EuroSCORE). Eur J Cardiothorac Surg 16, 9-13

O'Donoghue M, Braunwald E (2010): Natriuretic peptides in heart failure: should therapy be guided by BNP levels? Nat Rev Cardiol $\underline{7}, 13-20$

Otto CM (2001): Clinical practice. Evaluation and management of chronic mitral regurgitation. N Engl J Med $\underline{345}, 740-746$

Quittan M, Wiesinger GF, Crevenna R, Nuhr MJ, Posch M, Hulsmann M, Muller D, Pacher R, Fialka-Moser V (2001): Cross-cultural adaptation of the Minnesota Living with Heart Failure Questionnaire for German-speaking patients. J Rehabil Med $\underline{33}, 182-186$

Rector TS, Cohn JN (1992): Assessment of patient outcome with the Minnesota Living with Heart Failure questionnaire: reliability and validity during a randomized, double-blind, placebo-controlled trial of pimobendan. Pimobendan Multicenter Research Group. Am Heart J 124, 1017-1025

Redelmeier DA, Bayoumi AM, Goldstein RS, Guyatt GH (1997): Interpreting small differences in functional status: the Six Minute Walk test in chronic lung disease patients. Am J Respir Crit Care Med $\underline{155}, 1278-1282$

Rogers JH, Yeo KK, Carroll JD, Cleveland J, Reece TB, Gillinov AM, Rodriguez L, Whitlow P, Woo YJ, Herrmann HC, et al. (2009): Late surgical mitral valve repair after percutaneous repair with the MitraClip system. J Card Surg 24, 677-681

Roques F, Nashef SA, Michel P, Gauducheau E, de Vincentiis C, Baudet E, Cortina J, David M, Faichney A, Gabrielle F, et al. (1999): Risk factors and outcome in European cardiac surgery: analysis of the EuroSCORE multinational database of 19030 patients. Eur J Cardiothorac Surg 15, 816-822; discussion $822-813$

Roques F, Michel P, Goldstone AR, Nashef SA (2003): The logistic EuroSCORE. Eur Heart J 24, 881-882

Rosen SE, Borer JS, Hochreiter C, Supino P, Roman MJ, Devereux RB, Kligfield P, Bucek J (1994): Natural history of the asymptomatic/minimally symptomatic patient with severe mitral regurgitation secondary to mitral valve prolapse and normal right and left ventricular performance. Am J Cardiol $\underline{74}, 374-380$

STS-Database (1997): Mitral Valve Repair and Replacement Patients: Incidence of Complications Summary. (www.sts.org) 
Swaans MJ, Van den Branden BJ, Van der Heyden JA, Post MC, Rensing BJ, Eefting FD, Plokker HW, Jaarsma W (2009): Three-dimensional transoesophageal echocardiography in a patient undergoing percutaneous mitral valve repair using the edge-to-edge clip technique. Eur J Echocardiogr 10, 982-983

Tamburino C, Ussia GP, Maisano F, Capodanno D, La Canna G, Scandura S, Colombo A, Giacomini A, Michev I, Mangiafico S, et al. (2010): Percutaneous mitral valve repair with the MitraClip system: acute results from a real world setting. Eur Heart J $\underline{31}$, 1382-1389

Totaro P, Tulumello E, Fellini P, Rambaldini M, La Canna G, Coletti G, Zogno M, Lorusso R (1999): Mitral valve repair for isolated prolapse of the anterior leaflet: an 11-year follow-up. Eur J Cardiothorac Surg $\underline{15}, 119-126$

Umana JP, Salehizadeh B, DeRose JJ, Jr., Nahar T, Lotvin A, Homma S, Oz MC (1998): "Bow-tie" mitral valve repair: an adjuvant technique for ischemic mitral regurgitation. Ann Thorac Surg 66, 1640-1646

Vahanian A, Baumgartner H, Bax J, Butchart E, Dion R, Filippatos G, Flachskampf F, Hall R, lung B, Kasprzak J, et al. (2007): Guidelines on the management of valvular heart disease: The Task Force on the Management of Valvular Heart Disease of the European Society of Cardiology. Eur Heart J 28, 230-268

Yusoff R, Clayton N, Keevil B, Morris J, Ray S (2006): Utility of plasma N-terminal brain natriuretic peptide as a marker of functional capacity in patients with chronic severe mitral regurgitation. Am J Cardiol 97, 1498-1501

Zoghbi WA, Enriquez-Sarano M, Foster E, Grayburn PA, Kraft CD, Levine RA, Nihoyannopoulos P, Otto CM, Quinones MA, Rakowski $\mathrm{H}$, et al. (2003): Recommendations for evaluation of the severity of native valvular regurgitation with two-dimensional and Doppler echocardiography. J Am Soc Echocardiogr 16, 777-802 


\section{Lebenslauf}

Am 16.12.1984 wurde ich in Hamburg geboren. Von August 1991 bis Juni 1995 besuchte ich die Grundschule Windmühlenweg, danach 9 Jahre das humanistische Gymnasium Christianeum und schloss meine Schullaufbahn im Juli 2004 mit dem Abitur in den Hauptfächern Biologie, Geschichte, Deutsch, Englisch und dem großen Latinum ab. In der Oberstufe des Gymnasiums besuchte ich in der Zeit von Januar 2002 bis August 2002 die private Hochschule St. Pauls School in Brisbane, Australien.

Daraufhin absolvierte ich vom November 2004 bis zum Dezember 2004 ein Pflegepraktikum in der Chirurgie im Marienkrankenhaus in Hamburg. Im Januar 2005 folgte eine Ausbildung zum staatlich anerkannten Rettungssanitäter beim Arbeiter-Samariter-Bund, welche ich im April 2005 erfolgreich abschloss.

Nach diesen Ausbildungsschritten begann ich im April 2005 mein Medizinstudium an der GeorgAugust-Universität in Göttingen und schloss nach dem 4. Semester den ersten Abschnitt der ärztlichen Prüfung ab. Im vierten Fachsemester arbeitete ich zusätzlich als Hilfswissenschaftler im Zentrum für Anatomie und Embryologie als Assistent der makroskopischen Anatomie und leitete eine Gruppe von 12 Studenten. Im klinischen Abschnitt des Medizinstudiums arbeitete ich neben dem Studium von Juli 2007 bis Juli 2010 als Sitzwache in der Universitätsmedizin Göttingen. Von September 2008 bis März 2009 war ich Empfänger eines Auslandsstipendiums und studierte ein Semester lang an der Universität Miguel Hernandez de Elche in San Juan, Spanien. Seit April 2009 bin ich Doktorand in der kardiologischen Abteilung der Universitätsmedizin Göttingen und befasse mich wissenschaftlich mit der kathetergestützten Mitralklappenreparatur.

Momentan befinde ich mich im Praktischen Jahr des Medizinstudiums. In meinem ersten Tertial des Praktischen Jahres arbeitete ich in der Kardiologie am Mount Sinai Medical Center, New York, USA und am Royal Chest Hospital in London, UK. Das darauf folgende zweite Tertial absolvierte ich in der Unfallchirurgie an der Universitätsmedizin Göttingen. Momentan absolviere ich mein letztes Tertial als Unterassistent in der Dermatologischen Klinik des Universitätsspitals Zürich. 